\title{
Sensitive Dependence of Optimal Network Dynamics on Network Structure
}

\author{
Takashi Nishikawa, ${ }^{1}$ Jie Sun, ${ }^{2}$ and Adilson E. Motter ${ }^{1}$ \\ ${ }^{1}$ Department of Physics and Astronomy, Northwestern University, Evanston, Illinois 60208, USA \\ and Northwestern Institute on Complex Systems, Northwestern University, Evanston, Illinois 60208, USA \\ ${ }^{2}$ Department of Mathematics, Clarkson University, Potsdam, New York 13699, USA, \\ Department of Physics, Clarkson University, Potsdam, New York 13699, USA, \\ Department of Computer Science, Clarkson University, Potsdam, New York 13699, USA, \\ and Clarkson Center for Complex Systems Science, Clarkson University, Potsdam, New York 13699, USA
} (Received 3 November 2016; revised manuscript received 29 June 2017; published 28 November 2017)

The relation between network structure and dynamics is determinant for the behavior of complex systems in numerous domains. An important long-standing problem concerns the properties of the networks that optimize the dynamics with respect to a given performance measure. Here, we show that such optimization can lead to sensitive dependence of the dynamics on the structure of the network. Specifically, using diffusively coupled systems as examples, we demonstrate that the stability of a dynamical state can exhibit sensitivity to unweighted structural perturbations (i.e., link removals and node additions) for undirected optimal networks and to weighted perturbations (i.e., small changes in link weights) for directed optimal networks. As mechanisms underlying this sensitivity, we identify discontinuous transitions occurring in the complement of undirected optimal networks and the prevalence of eigenvector degeneracy in directed optimal networks. These findings establish a unified characterization of networks optimized for dynamical stability, which we illustrate using Turing instability in activator-inhibitor systems, synchronization in power-grid networks, network diffusion, and several other network processes. Our results suggest that the network structure of a complex system operating near an optimum can potentially be finetuned for a significantly enhanced stability compared to what one might expect from simple extrapolation. On the other hand, they also suggest constraints on how close to the optimum the system can be in practice. Finally, the results have potential implications for biophysical networks, which have evolved under the competing pressures of optimizing fitness while remaining robust against perturbations.

DOI: 10.1103/PhysRevX.7.041044

Subject Areas: Complex Systems, Nonlinear Dynamics

\section{INTRODUCTION}

Building on the classical fields of graph theory, statistical physics, and nonlinear dynamics, as well as on the increasing availability of large-scale network data, the field of network dynamics has flourished over the past 15 years $[1,2]$. Much of the current effort in this area is driven by the premise that understanding the structure, the function, and the relation between the two will help explain the workings of natural systems and facilitate the design of engineered systems with expanded capability, optimized performance, and enhanced robustness. There have been extensive studies on this structure-dynamics relation [3-5] in a wide range of contexts, such as synchronization [6-16]; reaction, diffusion, and/or advection dynamics [17-20]; dynamical stability [21,22]; controllability [23,24]; and information flow [25,26]. Many of these studies have led to systematic methods for enhancing the dynamics

Published by the American Physical Society under the terms of the Creative Commons Attribution 4.0 International license. Further distribution of this work must maintain attribution to the author(s) and the published article's title, journal citation, and DOI. through network-structural modifications, with examples including network control [27-30] and synchronization enhancement [31-34], where the latter has been demonstrated in applications [35-37].

A fundamental question at the core of the structuredynamics relation is that of optimization: which network structures optimize the dynamics of the system for a given function and what are the properties of such networks? The significance of addressing this question is twofold. First, knowledge of the properties of optimized structures can inform system architecture design. For example, in power-grid networks, whose operation requires frequency synchronization among power generators, the structures that maximize synchronization stability could potentially be used to devise effective strategies for upgrading the system [38]. Second, the identification of the network structures that guarantee the best fitness of natural complex systems can provide insights into the mechanisms underlying their evolution. Examples of such systems include neuronal networks, whose (synaptic) connectivity structure is believed to have been optimized through evolution or learning for categorization tasks [39], synchronization efficiency [40], dynamical complexity [41,42], information transfer efficiency [42,43], and/or wiring cost [40]. The question of optimizing the 
network structure can be conceptualized as the problem of maximizing or minimizing a measure of dynamical stability, robustness, or performance over all possible configurations of links connecting the dynamical units.

Here, we demonstrate that optimized dynamics are often highly sensitive to perturbations applied to the structure of the network. For concreteness, we focus on optimizing the linear stability of desired dynamical states, over all networks with a given number of nodes and links. We consider network states in which the (possibly timedependent) states of the individual nodes are identical across the network, such as in consensus dynamics, synchronized periodic or chaotic oscillations, and states of equilibrium in diffusion processes. We establish conditions under which the stability is sensitive or nonsensitive to structural perturbations, depending on the class of networks and the nature of the perturbations considered, as summarized in Fig. 1. In particular, we show that optimized stability can exhibit sensitivity under different types of perturbations for directed and undirected networks:

(1) Sensitivity to link removals and node additions (unweighted perturbations) for undirected optimal networks in the limit of large network size (upper red box in Fig. 1).

We show that such sensitivity is observed for a class of optimal networks, which we refer to as Uniform Complete Multipartite (UCM) networks. The UCM networks are composed of node groups of equal sizes that are fully connected to each other but have no internal links. We prove that these networks are the only networks that achieve the maximum stability possible for a given number of nodes and links. The UCM networks are part of a larger class of networks, characterized as having the Minimum possible size of the largest Components in their Complement (MCC) among all networks with a given number of nodes and links. We provide a full analytical characterization of the MCC networks of arbitrary finite size and study their behavior as the network size approaches infinity.
(2) Sensitivity to changes in link weights (weighted perturbations) for finite-size directed optimal networks (lower red box in Fig. 1).

While specific examples can be found in the literature [44-47], no systematic study exists on general mechanisms and conditions for such sensitivity. Here, we provide such conditions in terms of the spectral degeneracy of the network by establishing the scaling relation between the stability and the perturbation size. These conditions imply that spectral degeneracy underlies sensitivity to link-weight perturbations. We expect this sensitivity to be observed in many applications since spectral degeneracy appears to be common in real networks [48]. Moreover, here we show that optimization tends to increase the incidence of spectral degeneracy, and we also show that the network exhibits approximately the same sensitivity even when the degeneracy (or the optimality) is only approximate.

In addition to these two cases of sensitivity, we have results on the absence of sensitivity in the other two cases (blue boxes in Fig. 1). We illustrate the implications of our results using a general class of diffusively coupled systems for which the network spectrum is shown to determine the stability and other aspects of the dynamics to be optimized. The specific cases we analyze include the rate of diffusion over networks, the critical threshold for Turing instability in networks of activator-inhibitor systems, and synchronization stability in power grids and in networks of chaotic oscillators.

The remainder of the article is organized as follows. We first define the class of network dynamics under consideration (Sec. II). We then present our results on the two types of sensitivity anticipated above (Secs. III and IV), followed by examples of physical systems exhibiting these types of sensitivity (Sec. V). We conclude with a discussion on further implications of our results (Sec. VI).

\section{NETWORK DYNAMICS CONSIDERED}

We aim to address a wide range of network dynamics in a unified way. For this purpose, we consider the dynamics of

\section{Sensitivity of optimal networks}

$\begin{array}{ccc}\text { Unweighted perturbations } & \text { Undirected networks } \\ \text { Link removal or node addition in large networks } & \begin{array}{c}\text { Sensitive } \\ \text { (Sec. III A 3) }\end{array} \\ \begin{array}{c}\text { Weighted perturbations } \\ \text { Small changes in link weights } \\ \text { (Sec. IV B 2) }\end{array}\end{array}$

FIG. 1. Directed and undirected networks optimized for the stability of the network dynamics can be sensitive to weighted and unweighted structural perturbations, respectively. The graphs schematically illustrate typical behavior for systems with sensitivity (in red boxes) and systems with no sensitivity (in blue boxes). Under weighted perturbations, we actually show that all undirected networks (including nonoptimal ones) are nonsensitive (lower blue box). 
networks of coupled dynamical units governed by the following general equation with pairwise interactions:

$$
\dot{\mathbf{x}}_{i}=\mathbf{F}\left(\mathbf{x}_{i}, \mathbf{H}_{i 1}\left(\mathbf{x}_{i}, \mathbf{x}_{1}\right), \ldots, \mathbf{H}_{i n}\left(\mathbf{x}_{i}, \mathbf{x}_{n}\right)\right),
$$

for $i=1, \ldots, n$, where $n$ is the number of dynamical units (nodes), $\mathbf{x}_{i}=\mathbf{x}_{i}(t)$ is the column vector of state variables for the $i$ th node at time $t$, and $\dot{\mathbf{x}}_{i}$ denotes the time derivative of $\mathbf{x}_{i}$. The function $\mathbf{F}\left(\mathbf{x}, \mathbf{y}_{1}, \ldots, \mathbf{y}_{n}\right)$ is generally nonlinear and describes how the dynamics of node $i$ are influenced by the other nodes through intermediate variables $\mathbf{y}_{j}=$ $\mathbf{H}_{i j}\left(\mathbf{x}_{i}, \mathbf{x}_{j}\right)$, where $\mathbf{y}_{j}=\mathbf{0}$ indicates no interaction. This means that the dynamics of an isolated node are described by $\dot{\mathbf{x}}=\mathbf{F}(\mathbf{x}, \mathbf{0}, \ldots, \mathbf{0})$. We assume that the dependence of $\mathbf{F}$ on $\mathbf{y}_{j}$ is the same for all $j$ (or more precisely, that $\mathbf{F}$ is invariant under any permutation of $\left.\mathbf{y}_{1}, \ldots, \mathbf{y}_{n}\right)$. Thus, the topology of the interaction network and the strength of individual pairwise coupling are not encoded in $\mathbf{F}$, but rather in the $(i, j)$ dependence of the coupling function $\mathbf{H}_{i j}$. This extends the framework introduced in Ref. [49] and can describe a wide range of dynamical processes on networks, including consensus protocol [50,51], diffusion over networks [1], emergence of Turing patterns in networked activator-inhibitor systems [52], relaxation in certain fluid networks [53], and synchronization of power generators [54], as well as other coupled identical and nonidentical oscillators [49,55-57]. Details on these examples can be found in Supplemental Material [58], Sec. S1.

For the class of systems described by Eq. (1), we consider network-homogeneous states given by

$$
\mathbf{x}_{1}(t)=\cdots=\mathbf{x}_{n}(t)=\mathbf{x}^{*}(t),
$$

where $\mathbf{x}^{*}$ satisfies the equation for an isolated node, $\dot{\mathbf{x}}^{*}=\mathbf{F}\left(\mathbf{x}^{*}, \mathbf{0}, \ldots, \mathbf{0}\right)$. Each of the example systems mentioned above exhibits such a state: uniform agreement in consensus protocols, synchronous dynamics in oscillator networks, uniform occupancy in network diffusion, uniform concentration in coupled activator-inhibitor systems, and the equilibrium state in the fluid networks. Note that certain nonhomogeneous states can also be represented using such a solution by changing the frame of reference (demonstrated for specific examples of nonuniform phaselocked states in power grids and phase oscillator networks in Supplemental Material [58], Sec. S1A).

To facilitate the stability analysis, we make two general assumptions on the nature of node-to-node interactions when the system is close to a network-homogeneous state. Assumption (A-1): The interactions are "diffusive," in the sense that the coupling strength between two nodes, $\mathbf{H}_{i j}(\mathbf{u}, \mathbf{v})$, is to first order proportional to the difference between their states, $\mathbf{v}-\mathbf{u}$. In particular, we assume that the coupling strength vanishes as the node states become equal. Assumption (A-2): There is a constant coupling matrix $A=\left(A_{i j}\right)$ encoding the structure of the network of interactions, in the sense that the proportionality coefficient (the "diffusion constant") in assumption (A-1) can be written as $A_{i j} \cdot \mathbf{G}(t)$, where the scalar $A_{i j}$ represents the strength of coupling from node $j$ to node $i$, and the matrixvalued function $\mathbf{G}(t)$ is independent of $i$ and $j$.

Under these assumptions, we define a stability function $\Lambda(\alpha)$ for each complex-valued parameter $\alpha$ (derivation presented in Appendix A), which captures the factors determining the stability of the network-homogeneous state but is independent of the network structure. This function, referred to as a master stability function in the literature, was originally derived for a general class of systems that is different from the one we consider here $[49,56]$. The influence of the network structure on the stability is only through the (possibly complex) eigenvalues of the Laplacian matrix $L$, defined by

$$
L_{i j}:=d_{i} \delta_{i j}-A_{i j}, \quad d_{i}:=\sum_{j=1}^{n} A_{i j} .
$$

Note that $L$ always has a null eigenvalue $\lambda_{1}=0$ associated with the eigenvector $(1, \ldots, 1)^{T}$, which corresponds to the mode of instability that does not affect the condition in Eq. (2). The maximum Lyapunov exponent measuring the stability of the network-homogeneous state is then given by

$$
\Lambda_{\max }:=\max _{j \geq 2} \Lambda\left(\lambda_{j}\right)
$$

That is, the state is stable if $\Lambda_{\max }<0$ and unstable if $\Lambda_{\max }>0$. In addition, $\left|\Lambda_{\max }\right|$ gives the asymptotic rate of exponential convergence or divergence.

As an example of stability optimization, we consider the following fundamental question:

For a given number of nodes representing dynamical units, and a given number of links with identical weights, what is the assignment of links that maximizes the rate of convergence to a network-homogeneous state?

In the context of this problem, we may assume $A_{i j}$ to be binary $\left(A_{i j}=0\right.$ or 1$)$ without loss of generality, since any link weight $\varepsilon \neq 1$ can be factored out of $A_{i j}$ (making $A_{i j}$ binary) and absorbed into $\mathbf{G}(t)$, which is then accounted for by the stability function $\Lambda(\alpha)$.

\section{SENSITIVITY TO UNWEIGHTED PERTURBATIONS}

In this section, we demonstrate the sensitivity of the convergence rate to link removal and node addition in optimal undirected networks (Sec. III A). We then show that such sensitivity is not possible for optimal directed networks (Sec. III B). 


\section{A. Undirected networks}

\section{Optimization problem}

For the class of networks with a fixed number of undirected links $m=\sum_{i} \sum_{j>i} A_{i j}$, we have the additional constraint that the matrix $A$ is symmetric. This constraint can arise from the symmetry of the physical processes underlying the interaction represented by a link, such as the diffusion of chemicals through a channel connecting reactor cells in a chemical reaction network. In this case, the maximization of the convergence rate can be succinctly formulated as the minimization of $\Lambda_{\max }$ :

$$
\begin{array}{ll}
\operatorname{minimize} & \Lambda_{\max }(A) \\
\text { subject to } & A_{i j} \in\{0,1\}, A_{i i}=0, A_{i j}=A_{j i}, \\
& \sum_{i} \sum_{j>i} A_{i j}=m .
\end{array}
$$

If the stability function $\Lambda(\alpha)$ is strictly decreasing on the real line $\{\alpha \in \mathbb{C} \mid \operatorname{Im}(\alpha)=0\}$ for $\operatorname{Re}(\alpha) \leq \bar{\lambda}:=2 m /(n-1)$ (which is satisfied in most cases, as detailed in Supplemental Material [58], Sec. S1), maximizing the convergence rate to the network-homogeneous state for undirected networks is equivalent to maximizing $\lambda_{2}$, the smallest eigenvalue excluding the null eigenvalue that exists for any networks. We note that the problem is also equivalent to minimizing a bound on the deviations from a network-homogeneous state in a class of networks of nonidentical oscillators [59]. There have been a number of previous studies [57,60-64] on the related (but different) problem of maximizing the eigenratio $\lambda_{2} / \lambda_{n}$, which measures the synchronizability of the network structure for networks of coupled chaotic oscillators.

The maximization of $\lambda_{2}$ is generally a challenging task, except for the following particular cases. For $m=n(n-1) / 2$, the only network with $n$ nodes and $m$ links is the complete graph, resulting in the (maximum) value $\lambda_{2}=n$. For $m=n-1$ (implying that the network is a tree), the maximum possible value of $\lambda_{2}=1$ is achieved if and only if the network has the star configuration [53]. For other values of $m$ (assuming $m \geq n-1$ to ensure that the network is connected), it is challenging even numerically, mainly because each $A_{i j}$ is constrained to be either 0 or 1 , which makes it a difficult nonconvex combinatorial optimization. The problem of maximizing $\lambda_{2}$ has been a subject of substantial interest in graph theory, with several notable results in the limit $n \rightarrow \infty$, assuming that each node in the network has the same degree and that this common degree is constant [65-67] or assuming a fixed maximum degree [68]. In contrast to these bounded-degree results, below we address the maximization of $\lambda_{2}$ in a different limit, $n \rightarrow \infty$, keeping the link density $\phi:=2 m /[n(n-1)]$ constant.

\section{Optimal networks: UCM and MCC}

Here, we define UCM and MCC networks, and then show that they provide analytical solutions of the optimization problem formulated in the previous section. To define these networks, we first introduce two general quantities that characterize connected component sizes. For a given $k$, let function $M(n, k)$ denote the maximum number of links allowed for any $n$-node network whose connected components have size $\leq k$. Given $m$, we define $k_{n, m}$ to be the smallest (necessarily positive) integer $k$ for which $m \leq M(n, k)$; i.e., $k_{n, m}$ is the minimum size of the largest connected components of any network with $n$ nodes and $m$ links. We also use the notion of graph complements $[32,69,70]$. For a given network with adjacency matrix $A$, its complement is defined as the network with the adjacency matrix $A^{c}$ given by

$$
A_{i j}^{c}=\left(1-A_{i j}\right)\left(1-\delta_{i j}\right) .
$$

With these definitions and notations, we now define an MCC network to be one whose largest connected component of the complement is of size $k_{n, m_{c}}$, where $m_{c}:=$ $n(n-1) / 2-m$ is the number of links in the complement.

To see how the definition of MCC networks relates to the maximization of $\lambda_{2}$, we note that the maximum Laplacian eigenvalue of any network is upper bounded by its largest component size (stated and proved as Proposition 4 in Supplemental Material [58], Sec. S2B). We also note that the nonzero Laplacian eigenvalues of a network and its complement are related through

$$
\lambda_{n-i+2}^{c}=n-\lambda_{i}, \quad i=2, \ldots, n,
$$

where we denote the Laplacian eigenvalues of the network as $0=\lambda_{1} \leq \lambda_{2} \leq \cdots \leq \lambda_{n}$ (noting that the symmetry of $A$ constrains them to be real), and those of the complement as $0=\lambda_{1}^{c}<\lambda_{2}^{c} \leq \cdots \leq \lambda_{n}^{c}$. Thus, the smaller the largest component size in the complement, the smaller we expect the eigenvalue $\lambda_{n}^{c}$ to be, which would imply larger $\lambda_{2}$ according to Eq. (7).

For special combinations of $n$ and $m$, namely, $n=k \ell$ and $m=k^{2} \ell(\ell-1) / 2$ with arbitrary positive integers $k$ and $\ell$, the complement of an MCC network necessarily consists of $\ell$ components, each fully connected and of size $k$ (stated and proved as Proposition 2 in Supplemental Material [58], Sec. S2A). We refer to this unique MCC network as the UCM network for the given $n$ and $m$. Translating the structure of its complement to that of the network itself, the UCM network can be characterized as the one in which (i) the nodes are divided into $\ell$ groups of equal size $k$ (uniform), (ii) all pairs of nodes from different groups are connected (complete), and (iii) no pair of nodes within the same group are connected (multipartite). Figure 2 shows examples of UCM and MCC networks. 
(a) UCM network $\left(\lambda_{2}=12\right)$

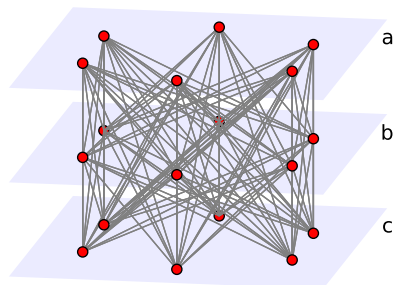

(c) Complement of UCM network

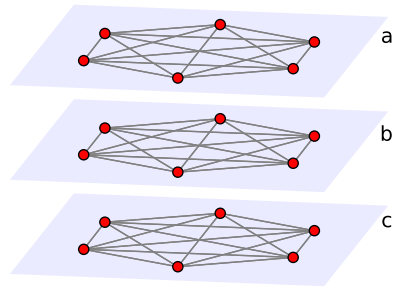

(b) MCC network $\left(\lambda_{2}=11\right)$

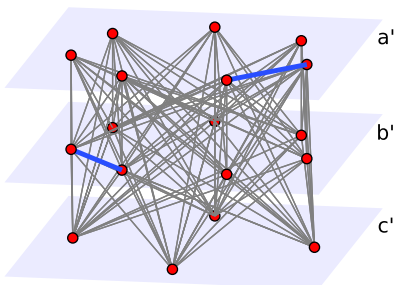

(d) Complement of MCC network

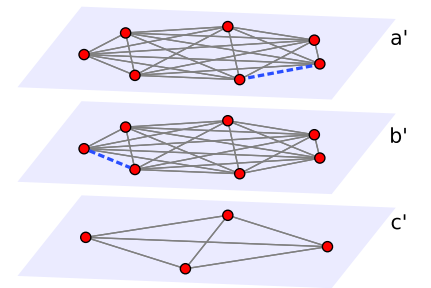

FIG. 2. UCM and MCC networks with $n=18$ nodes. (a) The UCM network with $\ell=3$ groups (labeled a, b, and c) of $k=6$ nodes each. All pairs of nodes belonging to different groups are connected, while all pairs within the same group are not connected, leading to a total of $m=k^{2} \ell(\ell-1) / 2=108$ links. (b) An MCC network constructed with the same number of nodes but with one fewer link $(m=107)$ and groups of unequal sizes (labeled $\mathrm{a}^{\prime}, \mathrm{b}^{\prime}$, and $\mathrm{c}^{\prime}$, and of sizes 7, 7, and 4, respectively). Note that in this case some nodes within the same group are connected (as indicated by solid blue lines). (c) The complement of the UCM network in (a), which has $\ell k(k-1) / 2=45$ links. In the complement, a node pair is connected if they are in the same group, and not connected if they are from different groups. (d) The complement of the MCC network in (b), which has 46 links. Since it has one more link than what can be accommodated by three isolated groups of size 6 [as in (c)], the minimum possible size of the largest component in the complement equals 7 in this case. Note that groups $\mathrm{a}^{\prime}$ and $\mathrm{b}^{\prime}$ have missing links (indicated by dashed blue lines), which correspond to the links within groups $\mathrm{a}^{\prime}$ and $\mathrm{b}^{\prime}$ in (b). The required increase in the size of the largest component in the complement forces $\lambda_{2}$ to decrease by one.

To establish the optimality of UCM and MCC networks, we first prove the following general upper bound:

$$
\lambda_{2} \leq\lfloor 2 m / n\rfloor=\lfloor\phi(n-1)\rfloor,
$$

for any $n$ and $m$ for which the link density $\phi=$ $2 m /[n(n-1)]<1$ (where $\lfloor x\rfloor$ denotes the largest integer not exceeding $x$ ). We prove this bound using Proposition 3.9.3 of Ref. [71], which states that

$$
\lambda_{n} \geq d_{\max }+1
$$

holds true for any network (with at least one link), where $d_{\max }$ denotes the maximum degree of the network. Applying this proposition to the complement of the network (rather than the network itself) gives

$$
\lambda_{n}^{c} \geq d_{\max }^{c}+1 \geq\left\lceil\bar{d}^{c}\right\rceil+1=\left\lceil 2 m_{c} / n\right\rceil+1,
$$

where $d_{\max }^{c}$ and $\bar{d}^{c}$ denote the maximum and mean degree of the complement, respectively, and $\lceil x\rceil$ denotes the smallest integer larger than or equal to $x$. Thus, we have $\lambda_{2}=$ $n-\lambda_{n}^{c} \leq n-\left(\left\lceil 2 m_{c} / n\right\rceil+1\right)=n-(\lceil n-1-2 m / n\rceil+1)=$ $\lfloor 2 m / n\rfloor$, establishing Eq. (8).

The optimality of UCM networks can now be established for any combination of $n$ and $m$ for which the UCM network can be defined [i.e., $n=k \ell$ and $m=k^{2} \ell(\ell-1) / 2$ ]. Indeed, since each connected component in the complement of such a UCM network is fully connected and of size $k$, it follows that the maximum Laplacian eigenvalue of the complement is $\lambda_{n}^{c}=k$. (This is because the Laplacian spectrum of a network is the union of the Laplacian spectra of its connected components, which is a known fact presented as Proposition 3 in Supplemental Material [58], Sec. S2B.) We thus conclude that $\lambda_{2}=n-k=\lfloor 2 m / n\rfloor$, implying that the UCM network attains the upper bound in Eq. (8) and has the maximum possible $\lambda_{2}$. Moreover, the UCM network is actually the only optimizer among all networks with the same $n$ and $m$ (proved in Appendix B).

For other MCC networks, we establish the formula

$$
\lambda_{2}=n-k_{n, m_{c}}
$$

for any link density $\phi<1$ and use it to show that MCC networks attain the upper bound in Eq. (8) and thus are optimal in several cases of lowest and highest link densities, as well as for a range of link density around each value corresponding to a UCM network. We also show that each MCC network is locally optimal in the space of all networks with the same $n$ and $m$ in the sense that $\lambda_{2} \leq$ $n-k_{n, m_{c}}$ holds true for any network obtained by rewiring a single link. Proofs of these results can be found in Supplemental Material [58], Secs. S2B and S2C. The optimality of these networks, which have fully connected clusters in the complement, suggests potential significance of other, more general network motifs [72], whose statistics have been studied in the context of network optimization $[73,74]$.

These $\lambda_{2}$-maximizing networks can be explicitly constructed. In fact, given any $n$ and $m$, an MCC network with $n$ nodes and $m$ links can be constructed by forming as many isolated, fully connected clusters of size $k_{n, m_{c}}$ as possible in the complement of the network. Details on this procedure are described in Appendix C, and a MATLAB implementation is available for download [75]. This procedure yields the (unique) UCM network if $n=k \ell$ and $m=k^{2} \ell(\ell-1) / 2$. Similar strategies that suppress the size of largest connected components, when incorporated into a network growth process, have been observed to cause discontinuous, or continuous but "explosive" percolation transitions [76-80]. The deterministic growth process defined in Ref. [81] is particularly close to the definition of MCC networks because the process explicitly minimizes 
the product of the sizes of the components connected by the new link in each step.

\section{Sensitivity of optimal networks}

To demonstrate the sensitivity of UCM networks to link removals and node additions, we first study the dependence of $\lambda_{2}$ for MCC networks on the link density $\phi<1$. By deriving an explicit formula for $k_{n, m_{c}}$, we rewrite Eq. (11) as

$$
\lambda_{2}=\left\lfloor C_{\ell, n}(\phi) \cdot n\right\rfloor
$$

where

$$
C_{\ell, n}(\phi)=\frac{\ell^{2}-\sqrt{\ell^{2}-\phi \ell(\ell+1)\left(1-\frac{1}{n}\right)}}{\ell(\ell+1)},
$$

and $\ell$ depends on $\phi$ and is defined as the unique integer satisfying

$$
1-\frac{1}{\ell} \leq\left(1-\frac{1}{n}\right) \phi<1-\frac{1}{\ell+1}
$$

(derivation presented in Supplemental Material [58], Sec. S2D). Equation (12) indicates that $\lambda_{2}$ experiences a series of sudden jumps as the link density increases from $\phi=2 / n$ (the minimum possible value for a connected network, corresponding to the star configuration) to $\phi=1$ (corresponding to the fully connected network). This behavior is better understood by considering the complement of the network as the number of links $m_{c}$ in the complement increases (corresponding to decreasing link density $\phi$ ), as illustrated for $n=20$ in Fig. 3. When the complement has exactly $M\left(n, k_{n, m_{c}}\right)$ links, any additional link would force the maximum component size $k_{n, m_{c}}$ to increase by one, causing a jump in $\lambda_{2}=n-k_{n, m_{c}}$. In Fig. 3, for example, when the network that has $m_{c}=$ $M(20,4)=30, k_{20,30}=4$, and $\lambda_{2}=16$ gains one more link in its complement $\left(m_{c}=31\right)$, the component size jumps to $k_{20,31}=5$ and $\lambda_{2}$ jumps down to 15 . The 18 -node UCM and MCC networks in Fig. 2 also illustrate such a jump. In the context of percolation problems, similar cascades of jumps in the maximum component size, called microtransition cascades, have been identified as precursors to global phase transitions [82].

Figure 4 demonstrates that for a wide range of $\phi$, the MCC networks improve $\lambda_{2}$ significantly over the ErdósRényi (ER) random networks, as well as those identified by direct numerical optimization of $\lambda_{2}$ using simulated annealing (SA). The difference is particularly large for $\phi$ near certain special values such as $1 / 2$. Note that the optimal value of $\lambda_{2}$ given by the upper bound (black curves) is achieved not only by the UCM network (for example, the one indicated by the green dot for $k=25, \ell=2)$ at $\phi=25 / 49 \approx 0.51$, but also by MCC networks (orange

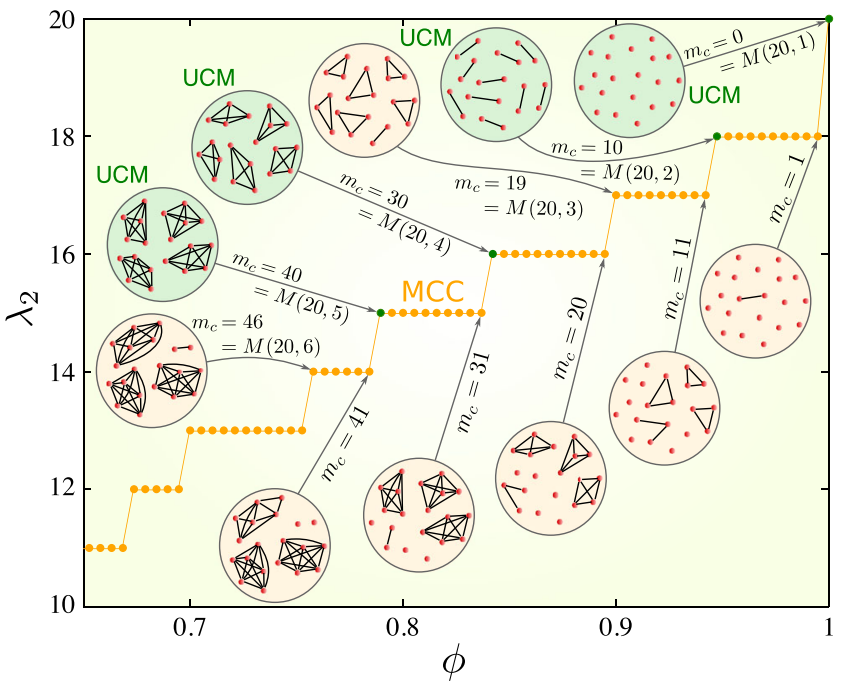

FIG. 3. UCM and MCC networks of size $n=20$ for the maximization of smallest nonzero Laplacian eigenvalue $\lambda_{2}$. For a given link density $\phi$, the orange dot indicates $\lambda_{2}$ for MCC networks. A UCM network, when possible for that value of $\phi$, is indicated by a green dot. As $\phi$ increases, the eigenvalue $\lambda_{2}$ experiences discrete jumps, corresponding to sudden changes in the structure of the network. The changes in the link configuration of the network's complement, as well as the associated jumps in the size of their largest clusters, are illustrated in the circles. At $\phi$ values just above and below the jumps, the complement has $m_{c}=M\left(n, k_{n, m_{c}}\right)$ links and $m_{c}=$ $M\left(n, k_{n, m_{c}}\right)+1$ links, respectively.

curves) for a finite range of $\phi$ around this value. The optimal $\lambda_{2}$ value, however, is sensitive to changes in the link density $\phi$, and it departs quickly from its value at $\phi=25 / 49$ as $\phi$ moves away from $25 / 49$, particularly for $\phi<25 / 49$.

In fact, $\lambda_{2}$ has many points exhibiting such sensitivity, which becomes more prominent for larger networks and turns into a singularity as $n \rightarrow \infty$ with fixed $\phi$. To see this, we take the limit in Eq. (12) to obtain

$$
\lim _{n \rightarrow \infty} \frac{\lambda_{2}}{n}=\frac{\ell^{2}-\sqrt{\ell^{2}-\phi \ell(\ell+1)}}{\ell(\ell+1)}
$$

where $\ell$ is the unique integer determined by $\phi_{\ell} \leq$ $\phi<\phi_{\ell+1}$, where we define $\phi_{\ell}:=1-1 / \ell=(\ell-1) / \ell$ for any positive integer $\ell$. This function of $\phi$, shown in Fig. 4 (red curve), has a cusplike dependence on $\phi$ around $\phi=\phi_{\ell}$, at which it achieves the asymptotic upper bound $\lim _{n \rightarrow \infty} \lambda_{2} / n \leq \phi$ [which follows directly from Eq. (8)] and has a square-root singularity on the left; i.e., the derivative on the left diverges (while the derivative on the right equals $1 / 2$ ). This singularity is inherently different from the discrete jumps observed above for finite $n$. Indeed, as the network size increases, the size of the jumps and the distance between consecutive jumps both tend to zero (as in the microtransition cascades [82] in percolation problems). The function thus becomes increasingly closer to a 


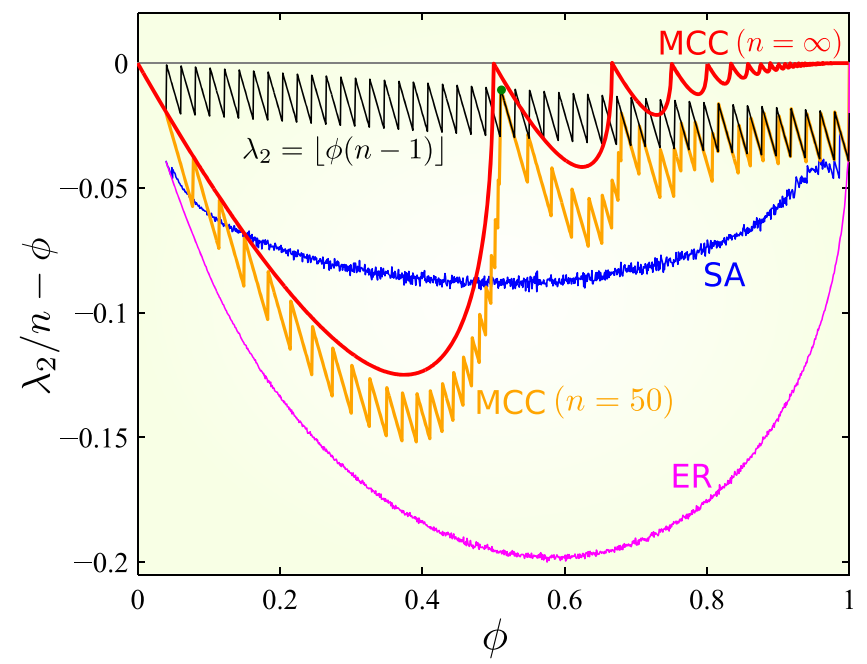

FIG. 4. Sensitive dependence of the Laplacian eigenvalue $\lambda_{2}$ on link density $\phi$ for undirected networks of size $n=50$. Each curve indicates $\lambda_{2}$ normalized by the network size $n$, relative to the link density $\phi$. (Plots of $\lambda_{2} / n$ itself can be found in Supplemental Material, Fig. S1 [58].) The blue curve shows the result of a single run of SA to maximize $\lambda_{2}$ with a fixed number of links. Each point on the magenta curve is the average over 1000 realizations of the ER random networks with connection probability $\phi$. The orange and red curves indicate the MCC networks for $n=50$ [Eq. (12)] and in the limit of $n \rightarrow \infty$ [Eq. (15)], respectively. Notice the square-root singularity on the left of points $\phi=\phi_{\ell}=(\ell-1) / \ell, \ell=2,3, \ldots$, on the red curve. The green dot near one of these singularity points indicates the UCM network with $\ell=2$ and $k=25$, which achieves the upper bound $\lambda_{2} \leq\lfloor\phi(n-1)\rfloor$ shown by the black curve.

(piecewise) smooth function, while the square-root singularity becomes progressively more visible (verified numerically in Fig. S2(a) of Supplemental Material [58]). For each singularity point $\phi=\phi_{\ell}$, there is a sequence of UCM networks with increasing $k$ (and thus increasing network size $n=k \ell)$, for which the link density $\phi=$ $(\ell-1) /(\ell-1 / k)$ approaches $\phi_{\ell}$ as $k \rightarrow \infty$.

The UCM networks associated with these singularities also exhibit sensitivity to the removal of an arbitrary link. As shown in the previous section, the UCM networks are the only networks that attain the upper bound in Eq. (8) and satisfy $\lambda_{2}=\lfloor 2 m / n\rfloor=\lfloor k(\ell-1)\rfloor=k(\ell-1)$ [where the last equality holds because $k(\ell-1)$ is an integer]. The removal of any single link reduces the bound to $\lfloor 2(m-1) / n\rfloor=\lfloor k(\ell-1)-2 / n\rfloor=k(\ell-1)-1$ and thus the normalized eigenvalue $\lambda_{2} / n-\phi$ by at least $1 / n$. Since the link removal reduces $\phi$ by $2 /[n(n-1)]$, the derivative of the normalized eigenvalue with respect to $\phi$ (in the limit of large $n$ ) is greater than or equal to

$$
\lim _{n \rightarrow \infty} \frac{1 / n}{2 /[n(n-1)]}=\lim _{n \rightarrow \infty} \frac{n-1}{2}=\infty .
$$

In terms of the complement, this can be understood as coming from the unavoidable increase of the component size, since the link removal in the network corresponds to a link addition in the complement. We note that the argument above is valid only for UCM networks, since the UCM network is the only one that attains the bound for any $\phi$ value at which the upper bound is discontinuous, i.e., when $2 m / n$ is an integer (proof given in Appendix B). In summary, we have the following result:

The UCM networks, which maximize $\lambda_{2}$ and correspond to singularities in the $\lambda_{2}$ vs $\phi$ curve for MCC networks, are sensitive to link removals.

The UCM networks show similar sensitivity to node additions as well. When $m$ is fixed, the expression for $\lambda_{2}$ given in Eq. (12), considered now as a function of $n$, has a square-root dependence on the right of the points $n=\sqrt{2 m / \phi_{\ell}}, \ell=2,3, .$. (corresponding to the UCM networks), as illustrated in Fig. S3 of Supplemental Material [58]. Similarly to the case of link removals, it can be shown that the bound in Eq. (8) suddenly drops from $k(\ell-1)$ to $k(\ell-1)-1$ when a new node is connected to the network as long as the number of new links is less than $m / n$, and that this drop leads to an infinite derivative for $\lambda_{2} / n-\phi$ with respect to $\phi$ in the limit of large $n$.

\section{B. Directed networks}

\section{Optimization problem}

For the class of networks with a fixed number of directed links $m_{d}=\sum_{i} \sum_{j \neq i} A_{i j}$, the matrix $A$ can be asymmetric in general. In this case, the problem of maximizing the rate of convergence to the network-homogeneous state can be expressed as

$$
\begin{array}{ll}
\operatorname{minimize} & \Lambda_{\max }(A) \\
\text { subject to } & A_{i j} \in\{0,1\}, \quad A_{i i}=0, \\
& \sum_{i} \sum_{j \neq i} A_{i j}=m_{d} .
\end{array}
$$

The solution of this problem generally depends on the specific shape of the stability function. However, the problem is equivalent to maximizing $\operatorname{Re}\left(\lambda_{2}\right)$, the smallest real part among the eigenvalues of $L$ excluding the identically null eigenvalue $\lambda_{1}$, if the stability function $\Lambda(\alpha)$ is strictly decreasing in $\operatorname{Re}(\alpha)$ and independent of $\operatorname{Im}(\alpha)$ for $\operatorname{Re}(\alpha) \leq \bar{\lambda}:=m_{d} /(n-1)$. This condition is satisfied, e.g., for consensus and diffusion processes (details presented in Supplemental Material [58], Secs. S1D and S1E, respectively). This equivalence is a consequence of the upper bound,

$$
\operatorname{Re}\left(\lambda_{2}\right) \leq \bar{\lambda}
$$


which follows from the fact that the sum of the eigenvalues equals the trace of $L$, which in turn equals $m_{d}$. [We note that the tighter bound in Eq. (8) is not applicable to directed networks in general.]

\section{Optimal networks}

The optimization problem just formulated can be solved if $m_{d}$ is "quantized," i.e., equals an integer multiple of $n-1$, in which case there are networks that satisfy $\lambda_{2}=\cdots=\lambda_{n}=\bar{\lambda}$ [32]. Such networks attain the upper bound in Eq. (18) and thus are optimal. The class of directed networks satisfying $\lambda_{2}=\cdots=\lambda_{n}=\bar{\lambda}$ has previously been studied within the context of network synchronization using objective functions that are not defined by $\operatorname{Re}\left(\lambda_{2}\right)$ and different from the convergence rate considered here [32,36]. If $m_{d}$ is not an integer multiple of $n-1$, the maximization of $\operatorname{Re}\left(\lambda_{2}\right)$, like the maximization of $\lambda_{2}$ for undirected networks, is a hard combinatorial optimization problem. Here, we compute the Laplacian eigenvalues symbolically (and thus exactly) for all directed networks of size $n=3,4$, and 5. For the quantized values of $m_{d}$, we verify that the upper bound $\bar{\lambda}$ is indeed attained [Figs. 5(a)-5(c)], in which case $\lambda_{2}=\cdots=\lambda_{n}=\bar{\lambda}$ is not
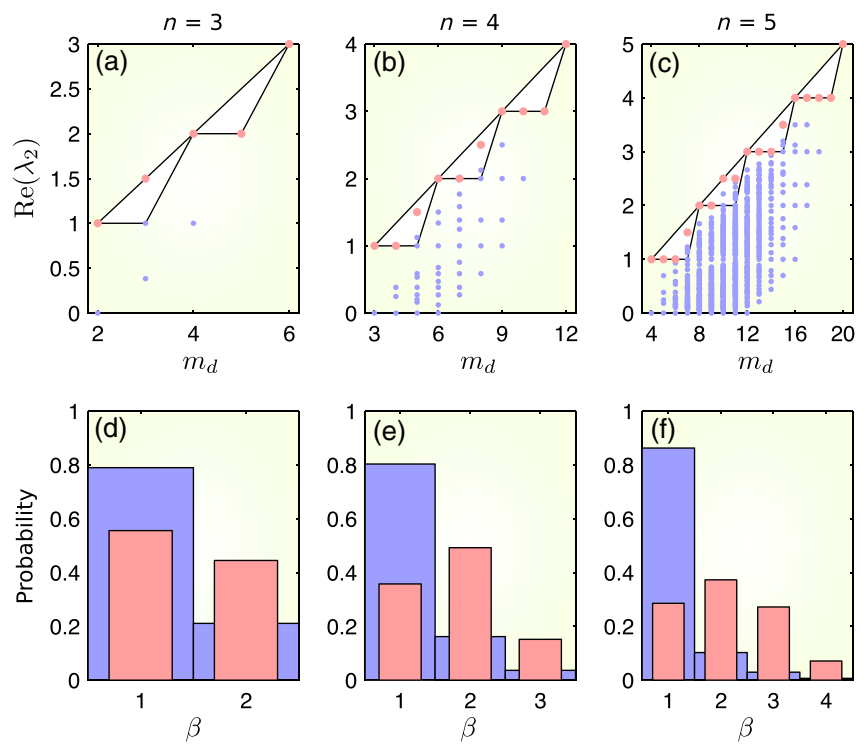

FIG. 5. Optimization induces spectral degeneracy. The maximum $\operatorname{Re}\left(\lambda_{2}\right)$ is computed exactly through symbolic calculation of eigenvalues for all directed networks of size $n=3,4$, and 5 . (a)-(c) Maximum values of $\operatorname{Re}\left(\lambda_{2}\right)$ as a function of the number of directed links, $m_{d}$ (red dots). All the other possible values of $\operatorname{Re}\left(\lambda_{2}\right)$ are indicated by blue dots. The black curves indicate the upper bound $\bar{\lambda}$ and lower bound $\lfloor\bar{\lambda}\rfloor$ for the maximum $\operatorname{Re}\left(\lambda_{2}\right)$. The upper bound comes from Eq. (18), while the lower bound is derived in Sec. III B 3. (d)-(f) Probability distribution of geometric degeneracy $\beta$ for $\lambda_{2}$ of all networks of a given size (blue bars) and of the $\operatorname{Re}\left(\lambda_{2}\right)$-maximizing networks (red bars). For each network size, the difference between the two distributions shows that the maximization tends to increase $\beta$. only real but also an integer. For intermediate values of $m_{d}$, the maximum $\operatorname{Re}\left(\lambda_{2}\right)$ does not appear to follow a simple rule; it can be strictly less than $\bar{\lambda}$, have nonzero imaginary part, and/or be noninteger.

\section{Nonsensitivity of optimal networks}

In the limit of large networks, however, there is a simple rule: we show below that the maximum value of $\operatorname{Re}\left(\lambda_{2}\right)$, normalized by $n$, converges to the link density $\phi:=$ $m_{d} /[n(n-1)]$ as $n \rightarrow \infty$ with $\phi$ fixed. This in particular implies that the normalized maximum $\operatorname{Re}\left(\lambda_{2}\right)$ has no sensitive dependence on $\phi$, in sharp contrast to the sensitivity observed in the same limit for undirected networks [83].

To establish this nonsensitivity result, we first note that $\phi=\bar{\lambda} / n$ is an upper bound for the maximum value of $\operatorname{Re}\left(\lambda_{2}\right) / n$, which follows immediately from Eq. (18). We show that the maximum value approaches the upper bound by showing that there is a lower bound that approaches the upper bound. The lower bound is established by constructing a specific network with $n$ nodes and $m_{d}$ directed links. To construct this network, we start with a variant of directed star networks, in which a core of $s$ fully connected nodes are all connected to all the other nodes, where we define $s:=[\bar{\lambda}]$. Since such a network involves exactly $s(n-1)$ links, the remaining $r$ links, where $r:=m_{d}-s(n-1)$, are added to the network. The network can thus be constructed as follows. (1) For each $i=1, \ldots, s$, add $n-1$ links from node $i$ to all the other nodes. (2) Add $r$ links from node $s+1$ to nodes $1, \ldots, r$ if $r \leq s$ and nodes $1, \ldots, s, s+2, \ldots, r+1$ if $r>s$. This network satisfies $\lambda_{2}=s=[\bar{\lambda}\rfloor$ (proof given in Appendix D), which provides a lower bound for the maximum value of $\operatorname{Re}\left(\lambda_{2}\right)$. This lower bound, as well as the upper bound $\bar{\lambda}$, is indicated by black curves in Figs. 5(a)-5(c) for $3 \leq n \leq 5$. Thus, the maximum value of $\operatorname{Re}\left(\lambda_{2}\right) / n$ is at least $s / n$, and this lower bound approaches the upper bound for large networks: $s / n=\lfloor\bar{\lambda}\rfloor / n=\lfloor\phi n\rfloor / n \rightarrow \phi$ as $n \rightarrow \infty$. This proves our claim that $\operatorname{Re}\left(\lambda_{2}\right) / n$ for optimal networks is a smooth function of $\phi$ in the limit of large networks, thus establishing the absence of sensitivity.

\section{SENSITIVITY TO WEIGHTED PERTURBATIONS}

To demonstrate the second type of sensitivity, we now study how the convergence rate behaves when a small weighted perturbation is applied to the network structure, particularly when the initial network is optimal or close to being optimal. Since the convergence rate is determined by the Laplacian eigenvalues through the stability function $\Lambda(\alpha)$ and Eq. (4), it suffices to analyze how the Laplacian eigenvalues respond to such perturbations, which we formulate as perturbations of the adjacency matrix in the form $A+\delta \Delta A$, where the small parameter $\delta$ is positive (unless noted otherwise) and $\Delta A$ is a fixed matrix. This type of structural perturbations can represent imperfections in the strengths of couplings in real networks, such as power grids 
and networks of chemical [84], electrochemical [85], or optoelectronic [36] oscillators.

\section{A. Eigenvalue scaling for arbitrary networks}

Here, we show that for a given Laplacian eigenvalue $\lambda$ of a directed network and a generic choice of $\Delta A$, the change $\Delta \lambda$ of the eigenvalue due to the perturbation generally follows a scaling relation, $|\Delta \lambda| \sim \delta^{\gamma}$. We also provide a rigorous bound for the scaling exponent $\gamma$. This scaling exponent determines the nature of the dependence of the perturbed eigenvalue on $\delta$ : if $0<\gamma<1$, the dependence is sensitive and characterized by an infinite derivative at $\delta=0$, and if $\gamma \geq 1$, it is nonsensitive and characterized by a finite derivative.

\section{Bound on scaling exponent}

We provide an informative bound on $\gamma$ by proving the following general result on matrix perturbations. Suppose $\lambda$ is an eigenvalue of an arbitrary matrix $M$ with geometric degeneracy $\beta$ [36], defined as the largest number of repetitions of $\lambda$ associated with the same eigenvector (i.e., the size of the largest diagonal block associated with $\lambda$ in the Jordan canonical form of $M$ ). For perturbations of the form $M+\delta \Delta M$ with an arbitrary matrix $\Delta M$, there exists a constant $C$ such that the corresponding change $\Delta \lambda=\Delta \lambda(\delta)$ in the eigenvalue, as a function of $\delta$, satisfies

$$
\underset{\delta \rightarrow 0}{\limsup } \frac{|\Delta \lambda(\delta)|}{\delta^{1 / \beta}} \leq C
$$

(proof given in Appendix E). Applying this result to an eigenvalue $\lambda$ of the Laplacian matrix $L$, we see that $\gamma \geq 1 / \beta$, implying that the set of perturbed eigenvalues that converge to $\lambda$ as $\delta \rightarrow 0$ do so at a rate no slower than $\delta^{1 / \beta}$.

\section{Typical scaling behavior}

The bound established above suggests that the scaling $|\Delta \lambda| \sim \delta^{1 / \beta}$ would be observed for all perturbed eigenvalues that converge to $\lambda$ as $\delta \rightarrow 0$. In fact, our numerics supports a more refined statement for networks under generic weighted structural perturbations: for each eigenvector (say, the $j$ th one) associated with $\lambda$, there is a set of $\beta_{j}$ perturbed eigenvalues that converge to $\lambda$ as $\delta \rightarrow 0$ and follows the scaling,

$$
|\Delta \lambda| \sim \delta^{1 / \beta_{j}}
$$

where $\beta_{j}$ is the number of repetitions of $\lambda$ associated with the $j$ th eigenvector (i.e., the size of the $j$ th Jordan block associated with $\lambda$ ). We numerically verify this individual scaling for Laplacian eigenvalues using random perturbations applied to all off-diagonal elements of $A$. We consider two examples of directed networks of size $n=6$, shown at the top of Fig. 6, which are both optimal because $\lambda_{2}=\cdots=\lambda_{6}$. For each of these networks, the plots on the left-hand side of the corresponding panel of Fig. 6 show the distributions of the scaling exponent $\gamma$ in the relation $|\Delta \lambda| \sim \delta^{\gamma}$ for random choices of $\Delta A$, where $\gamma$ is estimated from fitting the computed values of perturbed $\lambda_{i}$ over different ranges of $\delta$. We see that the distributions are sharply peaked around $\beta_{j}$ (indicated by the gray inverted triangles) with smaller spread for narrower ranges of $\delta$, supporting the asymptotic scaling in Eq. (20) in the limit $\delta \rightarrow 0$.

We note that, for nongeneric weighted perturbations (e.g., if the perturbation is constrained to a subset of the offdiagonal elements of $A$ ), the exponent may be different from $1 / \beta_{j}$ in Eq. (20). For example, when perturbing only the existing links of a directed tree (which is optimal with $\lambda_{2}=\cdots=\lambda_{n}=1$ ), the exponent is one, and thus the network is not sensitive to this type of perturbations even if the degeneracy $\beta>1$, as illustrated in Fig. 6(a) (plots on the right-hand side). This follows from the fact that the Laplacian matrix of a directed tree is triangular under appropriate indexing of its nodes, which remains true after perturbing the existing links. This nonsensitivity result can be extended to certain other cases, e.g., when $P^{-1} \Delta L P$ is a triangular matrix, where $P$ is the nonsingular matrix in the Jordan decomposition of $L$ and $\Delta L$ is the perturbation of the Laplacian matrix (proof presented in Appendix F). In other cases, the scaling with exponent $1 / \beta_{j}$, as in Eq. (20), can be observed even when perturbing only the existing links, as illustrated in Fig. 6(b) (plots on the right-hand side).

\section{B. Classification of networks by their sensitivity}

The general scaling results in the previous section indicate that the overall sensitivity of a Laplacian eigenvalue is determined by its geometric degeneracy $\beta$. This is because larger $\beta_{j}$ means more sensitive dependence on $\delta$ in Eq. (20) and because $\beta$ is by definition the largest among all the associated $\beta_{j}$ 's. Thus, we summarize as follows:

A Laplacian eigenvalue is sensitive to generic weighted perturbations if and only if the geometric degeneracy $\beta>1$, i.e., the associated eigenvector is degenerate.

\section{Sensitivity in directed networks}

We now show that optimal directed networks are often sensitive to generic weighted perturbations. Figure 7 shows examples from the class of optimal networks satisfying $\lambda_{2}=\cdots=\lambda_{n}$. The geometric degeneracy $\beta$ can be different for different optimal networks in this class and provides a measure of how sensitive an eigenvalue is when $\beta>1$. Some of these networks are nonsensitive, including simple cases such as the fully connected networks and directed star networks, as well as other networks with more complicated structure, such as the network $G_{1}$ in Fig. 7. Other optimal 
(a)

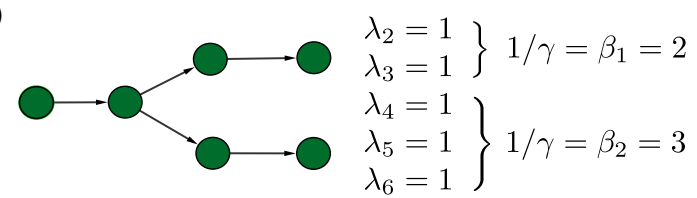

Perturbing all off-diagonal $A_{i j}$
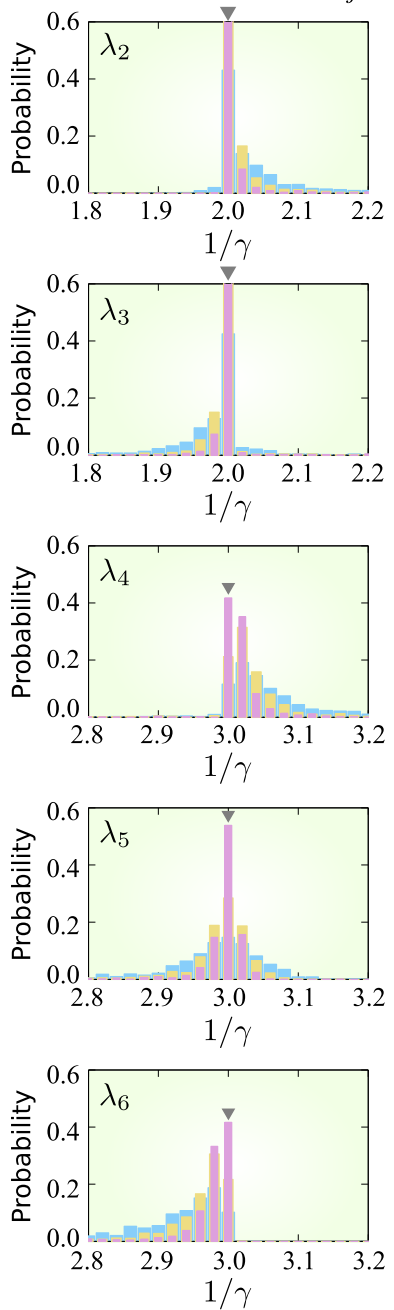

Perturbing existing links only
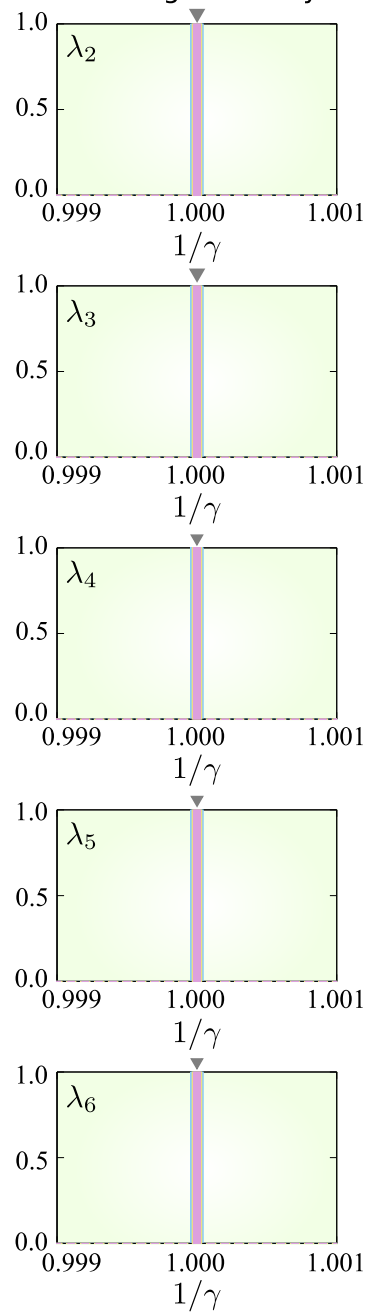

(b)

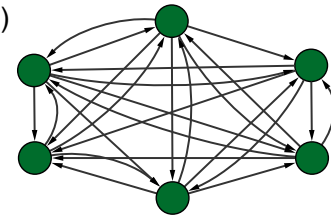

Perturbing all off-diagonal $A_{i j}$
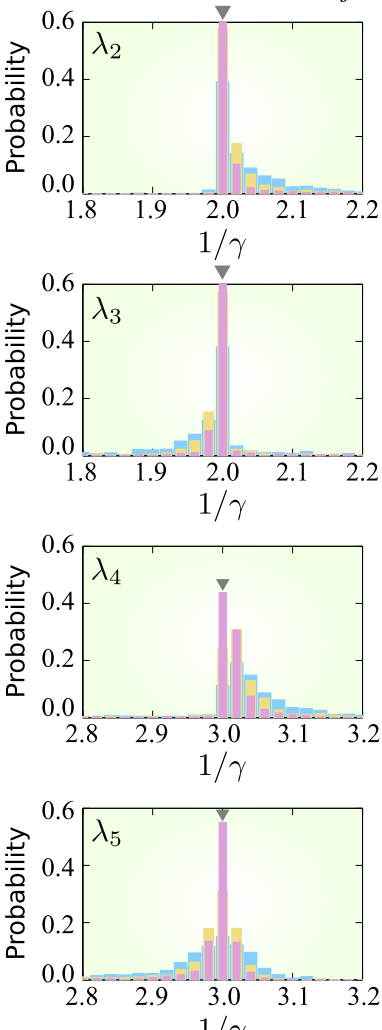

$1 / \gamma$

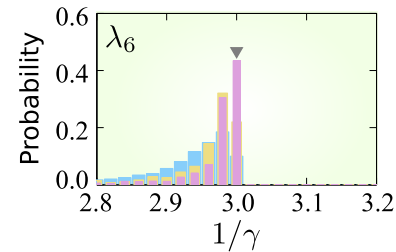

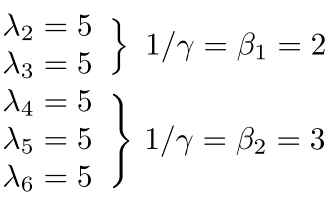

Perturbing existing links only
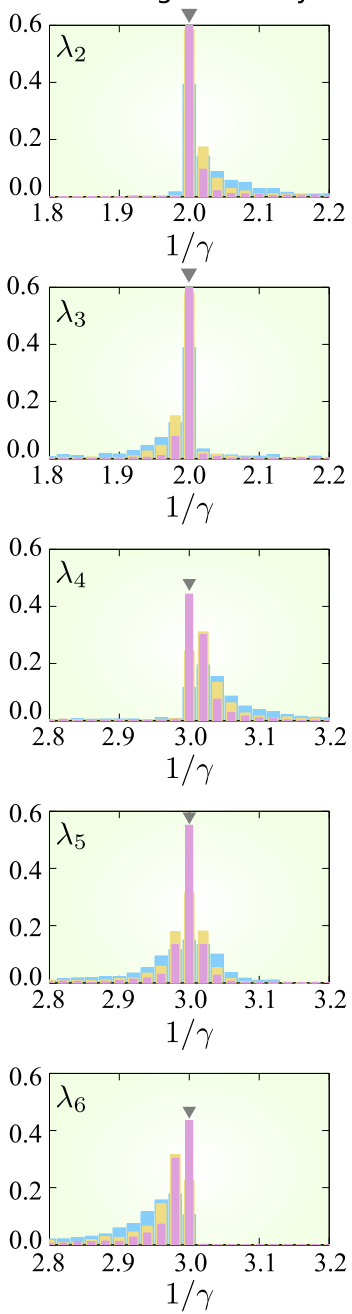

FIG. 6. Distribution of scaling exponents for the Laplacian eigenvalues of 6-node optimal directed networks under random structural perturbations. (a) Example network (a directed tree) with $\lambda_{2}=\cdots=\lambda_{6}=1$. (b) Example network with $\lambda_{2}=\cdots=\lambda_{6}=5$. Each plot shows histograms of $1 / \gamma$, where $\gamma$ is the scaling exponent numerically estimated from $\lambda_{i}$ (the $i$ th smallest perturbed eigenvalue) computed at 1000 equally spaced values of $\delta$ in the intervals $\left[0,10^{-3}\right]$ (blue), $\left[0,10^{-4}\right]$ (yellow), and $\left[0,10^{-5}\right]$ (pink). We determine $\gamma$ by applying MATLAB's built-in linear least-squares algorithm in log scale [86]. Each histogram is generated by estimating $\gamma$ for 10000 realizations of $\Delta A$, where each element of $\Delta A$ (corresponding to the perturbation of the weight of an existing link or the addition of a new link with small weight) is chosen randomly from the uniform distribution on $[-1,1]$. When perturbing all the off-diagonal elements of the adjacency matrix $A$ (plots on the left-hand side of each panel), the results support $1 / \gamma=\beta_{j}$ for both networks. When perturbing only the existing links (plots on the right-hand side of each panel), the scaling exponent depends on the initial network: the plots support $1 / \gamma=1$ for the directed tree in (a) and $1 / \gamma=\beta_{j}$ for the network in (b).

networks in this class are sensitive, and there is a hierarchy of networks having different levels of sensitivity, from $\beta=2$ (e.g., network $G_{2}$ in Fig. 7) all the way up to the maximum possible value $\beta=n-1$ (e.g., network $G_{19}$ in Fig. 7), including all intermediate cases (e.g., network $G_{7}$ in Fig. 7). Such scaling behavior and the resulting sensitivity for $\beta>1$ are robust in the sense that they would be observed even if the associated eigenvector is only approximately degenerate (proved in Appendix G).

How often does an optimal network (including those not satisfying $\lambda_{2}=\cdots=\lambda_{n}$ ) have $\beta>1$ and thus exhibit sensitivity? To study this systematically, we compute $\beta_{j}$ 


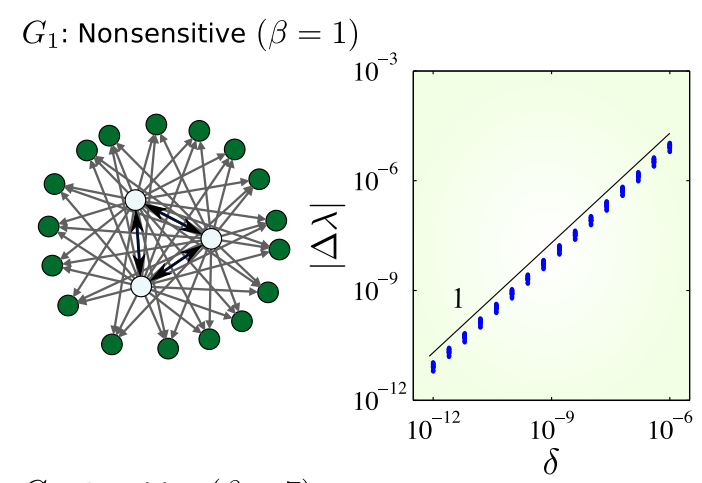

$$
G_{2}: \text { Sensitive }(\beta=2)
$$
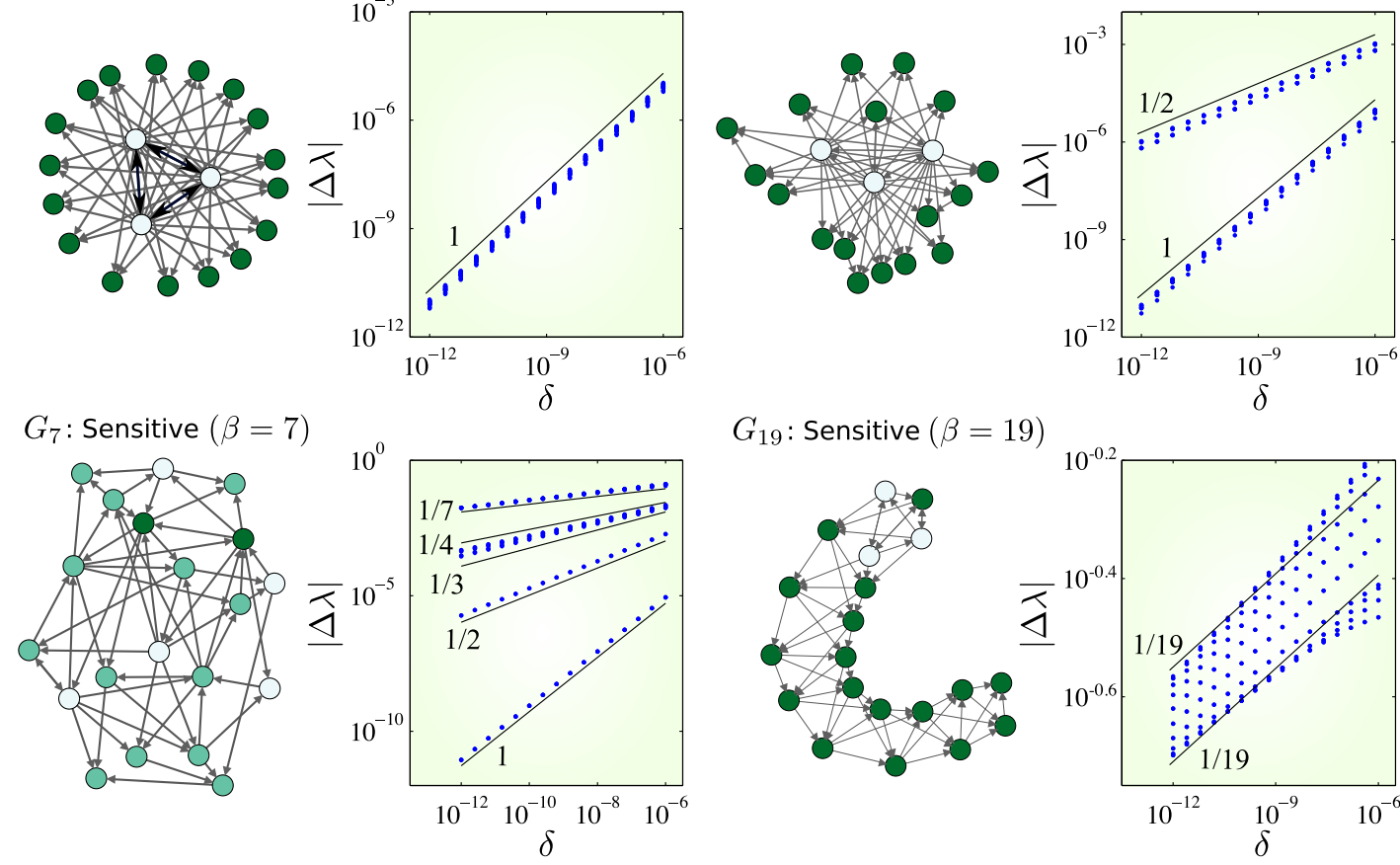

$G_{19}$ : Sensitive $(\beta=19)$

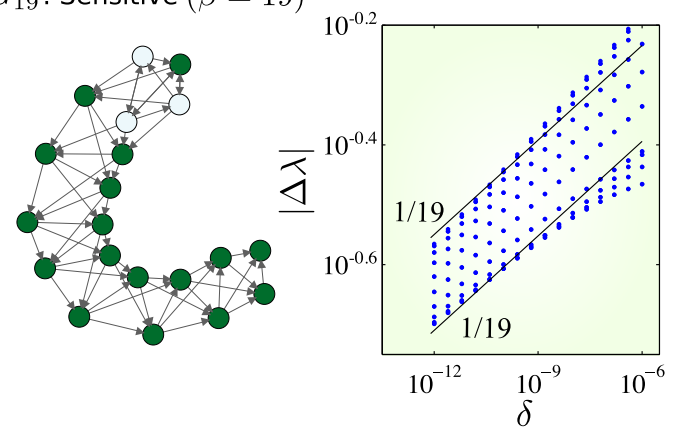

FIG. 7. Optimal directed networks with various levels of sensitivity to generic weighted perturbations. Example networks $G_{1}, G_{2}, G_{7}$, and $G_{19}$ (each with 20 nodes and 57 links) all satisfy $\lambda_{2}=\cdots=\lambda_{20}=3$ (and thus are optimal) but have different geometric degeneracy. Perturbing the adjacency matrix of each network as $A+\delta \Delta A$, we plot in double logarithmic scale the resulting change $|\Delta \lambda(\delta)|=$ $|\lambda(\delta)-3|$ for all 19 Laplacian eigenvalues (blue dots, many of which are overlapping). The same randomly chosen $\Delta A$ is used for all four networks, where each $\Delta A_{i j}$ is drawn uniformly from the interval $[-1,1]$ if the link exists from node $j$ to node $i$, and from $[0,1]$ otherwise. The perturbations thus allow small increase and decrease of the weight of existing links, as well as the addition of new links with small weight. In each network, nodes of the same color indicates the same in-degree, and a bidirectional arrow represents two directed links in opposite directions. The three nodes in the center of $G_{1}$ form a fully connected triangle and each of the other nodes has three in-links from these center nodes. In each plot, we observe the expected scaling behavior in Eq. (20), indicated by black lines, each labeled with the corresponding scaling exponent, $1 / \beta_{j}\left(G_{1}: \beta=1\right.$ with 19 eigenvectors and $\beta_{1}=\cdots=\beta_{19}=1 ; G_{2}: \beta=2$ with 15 eigenvectors and $\beta_{1}=\cdots=\beta_{11}=1, \beta_{12}=\cdots=\beta_{15}=2 ; G_{7}: \beta=7$ with 7 eigenvectors and $\beta_{1}=\beta_{2}=\beta_{3}=1, \beta_{4}=2, \beta_{5}=3$, $\beta_{6}=4, \beta_{7}=7 ; G_{19}: \beta=19$ with 1 eigenvector and $\left.\beta_{1}=19\right)$.

symbolically and thus exactly for each Laplacian eigenvalue of all possible directed networks with $n \leq 5$. We find that a large fraction of the $\operatorname{Re}\left(\lambda_{2}\right)$-maximizing networks are indeed sensitive due to geometric degeneracy: $44.4 \%, 64.3 \%$, and $71.5 \%$ of them have $\beta>1$ for $n=3$, 4 , and 5, respectively [red bars in Figs. 5(d)-5(f)]. These fractions are significantly higher than the corresponding fractions among all directed networks (including nonoptimal ones): $21.1 \%, 19.7 \%$, and $13.7 \%$, respectively [blue bars in Figs. 5(d)-5(f)]. Since $\beta$ is bounded by the algebraic degeneracy (multiplicity) of $\lambda_{2}$, an interesting question is to ask how often $\beta$ attains this bound, giving the network the maximum possible level of sensitivity. Among those networks that are both optimal and sensitive, $74.5 \%$ and $60.0 \%$ achieve the maximal sensitivity for $n=4$ and 5, respectively. (The fraction is trivially $100 \%$ for $n=3$.) These results thus suggest that optimal directed networks are much more likely to exhibit higher sensitivity than nonoptimal ones.

\section{Nonsensitivity in undirected networks}

The situation is drastically different when the network is undirected. For an arbitrary undirected network, for which we have the constraint that the matrix $A$ is symmetric, all of its Laplacian eigenvalues are nonsensitive to any (generic or nongeneric) perturbation of the form $A+\delta \Delta A$, since symmetric matrices are diagonalizable [87] and thus $\gamma \geq 1 / \beta=1$. This in particular implies that there is no sensitivity even for optimal undirected networks, including the UCM and MCC networks. However, this is not in contradiction with the results in Sec. III A, as they concern finitesize perturbations (i.e., addition or removal of whole links) in the limit of large networks, while here we consider infinitesimal perturbations on link weights for finite-size networks.

\section{Generality of the scaling}

The scaling bound in Eq. (19) is applicable to both directed and undirected networks, regardless of whether the 
links are weighted or unweighted. We also expect the scaling in Eq. (20) to generically hold true across these classes of networks. Moreover, while the results for unweighted perturbations in Sec. III are specific to the Laplacian eigenvalue $\lambda_{2}$, Eq. (19) applies to any eigenvalue of an arbitrary matrix, including the adjacency matrix and any other matrix that may characterize a particular system. For example, the largest eigenvalue (in absolute value) of the adjacency matrix for a strongly connected (directed) network is nondegenerate (by, e.g., the Perron-Frobenious theorem [88]) and therefore nonsensitive. In general, the degree to which the scaling holds is likely to be related to the normality of the matrix, which can range from completely normal matrices with orthogonal eigenvectors (as in undirected networks) to highly non-normal matrices with parallel, degenerate eigenvectors (as in many optimal networks) [89,90]. The result in Appendix G implies that the network does not need to be perfectly degenerate, which opens the door for observing the sensitivity we identified in real-world applications where exact degeneracy is unlikely [36]. Combining all these with the tendency of optimization to cause geometric degeneracy and with the wide range of systems that can be described by Eq. (1), we expect to observe sensitivity to weighted perturbations in many applications.

\section{SENSITIVITY IN EXAMPLE PHYSICAL SYSTEMS}

As summarized in Fig. 1, we established two cases in which sensitive dependence on network structure arises: undirected networks under unweighted perturbations (Sec. III A 3) and directed networks under weighted perturbations (Sec. IV B 1). Here, we discuss implications of these cases for concrete examples of physical networked systems.

\section{A. Undirected networks under unweighted perturbations}

For undirected networks, the sensitivity of $\lambda_{2}$ observed for UCM networks is relevant for a wide range of networked systems, since the stability function formalism establishes that, in many systems, $\lambda_{2}$ determines the stability properties of relevant network-homogeneous states. Typically the asymptotic rate of convergence $\left|\Lambda_{\max }\right|$ is a smooth, monotonically increasing function of $\lambda_{2}$ (concrete examples given in Supplemental Material [58], Secs. S1C-S1F), and thus the maximized convergence rate exhibits sensitivity. Below we list specific cases in which sensitivity is observed in $\left|\Lambda_{\max }\right|$ or a related quantity:

(1) Convergence rate. For networks of phase oscillators, including models of power-grid networks, the convergence rate to a frequency-synchronized, phaselocked state is a function of the Laplacian eigenvalue $\tilde{\lambda}_{2}$ associated with an effective interaction matrix $\tilde{A}$ for the system (details presented in Supplemental Material [58], Sec. S1A). While $\lambda_{2}$ is generally different from $\tilde{\lambda}_{2}$, it is strongly correlated with $\tilde{\lambda}_{2}$, and hence with $\Lambda_{\max }$. We thus expect to observe sensitive dependence of $\Lambda_{\max }$, which is indeed confirmed in Fig. 8(a) for power-grid networks with a prescribed network topology and realistic parameters for the generators and other electrical components in the system.

(2) Transient dynamics. In addition to the asymptotic convergence rate $\Lambda_{\max }$, sensitive dependence can be observed for the convergence rate in the transient
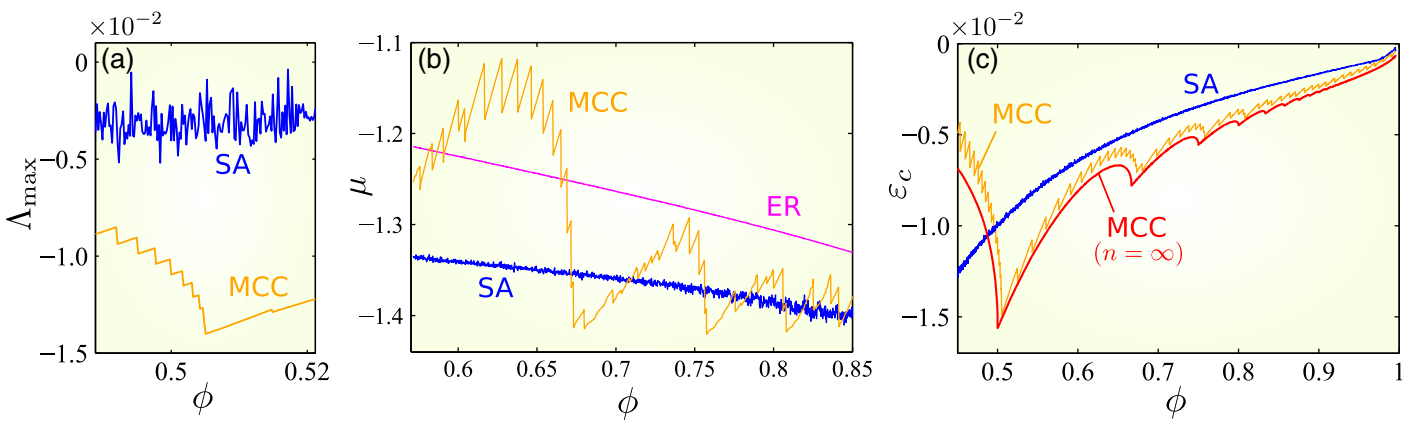

FIG. 8. Sensitive dependence on link density $\phi$ in physical examples of undirected networks. (a) Exponential rate of convergence $\Lambda_{\text {max }}$ to a synchronous state of power-grid networks. (b) Mean finite-time convergence rate $\mu$ toward synchronization in networks of optoelectronic oscillators. (c) Critical diffusivity threshold $\varepsilon_{c}$ for Turing instability in networks of activator-inhibitor systems. Description of these three systems can be found in Supplemental Material [58], Secs. S1A-S1C. The orange curves indicate the values of these quantities for the MCC networks with $n=100$ constructed by the procedure described in Appendix C, while the red curve in (c) is the finite- $n$ approximation obtained from the asymptotic formula in Eq. (15). The blue curves indicate the corresponding values for networks found by SA. The magenta curve in (b) is the mean value for the ER random networks estimated from 1000 realizations, while in (a) and (c) the values are relative to the corresponding mean value for the ER random networks (and thus zero corresponds to the ER mean value). 
dynamics of the network, which depends not only on $\lambda_{2}$ but on all Laplacian eigenvalues. This is illustrated in Fig. 8(b) using the example of coupled optoelectronic oscillator networks (system details described in Supplemental Material [58], Sec. S1B).

(3) Critical coupling threshold. Another physical quantity that can exhibit sensitive dependence is the critical coupling threshold for the stability of the network-homogeneous state in systems with a global coupling strength $\varepsilon$. In such systems, the functions $\mathbf{H}_{i j}$ are proportional to $\varepsilon$. For identical oscillators capable of chaotic synchronization, the minimum coupling strength for stable synchronization is inversely proportional to $\lambda_{2}$. For the activatorinhibitor systems [52], the parameter $\varepsilon$ is interpreted as the common diffusivity constant associated with the process of diffusion over individual links. As $\varepsilon$ is decreased from a value sufficiently large for the uniform concentration state to be stable, there is a critical diffusivity, $\varepsilon=\varepsilon_{c}$, corresponding to the onset of Turing instability. This $\varepsilon_{c}$ is inversely proportional to $\lambda_{2}$ (derivation given in Supplemental Material [58], Sec. S1C). Such a critical threshold thus depends sensitively on the link density of the network [as illustrated in Fig. 8(c)] as well as on the number of nodes.

\section{B. Directed networks under weighted perturbations}

For directed networks, the sensitivity of Laplacian eigenvalues under generic perturbations is typically inherited by the convergence rate $\Lambda_{\max }$ for many systems and processes governed by Eq. (1), including most of the examples described in Supplemental Material [58], Sec. S1. In fact, $\Lambda_{\max }$ would have the same sensitivity as the Laplacian eigenvalue $\lambda_{j}$ whenever $\Lambda_{\max }$ has a smooth (nonconstant) dependence on $\lambda_{j}$ near the unperturbed values of $\lambda_{j}$. Figure 9 illustrates the sharp contrast between sensitive and nonsensitive cases using the example of synchronization in networks of chaotic optoelectronic oscillators [36] (system details described in Supplemental Material [58], Sec. S1B).

\section{DISCUSSION}

The sensitive dependence of collective dynamics on the network structure, characterized here by a derivative that diverges at an optimal point, has several implications. On the one hand, it implies that the dynamics can be manipulated substantially by small structural adjustments, which we suggest has the potential to lead to new control approaches based on modifying the effective structure of the network in real time; indeed, the closer the system is to being optimal, the larger the range of manipulation possible with the same amount of structural adjustment. On the other hand, the observed cusplike behavior imposes constraints on how close one can get to the ultimate optimum in practice, given unavoidable parameter mismatches, resolution limits, and numerical uncertainty.

It is insightful to interpret our results in the context of living systems. The apparent conundrum that follows from this study is that biological networks (such as genetic, neuronal, and ecological ones) are believed to have evolved under the pressure to both optimize fitness and be robust to structural perturbations [91]. The latter means that the networks would not undergo significant loss of function (hence, of optimality) when perturbed. For example, a mutation in a bacterium (i.e., a structural change to a genetic network) causes the resulting strain to be nonviable in only a minority of cases [92]. A plausible explanation is that much of the robustness of living systems comes from the plasticity they acquire from optimizing their fitness under varying conditions $[93,94]$. In the case of bacterial
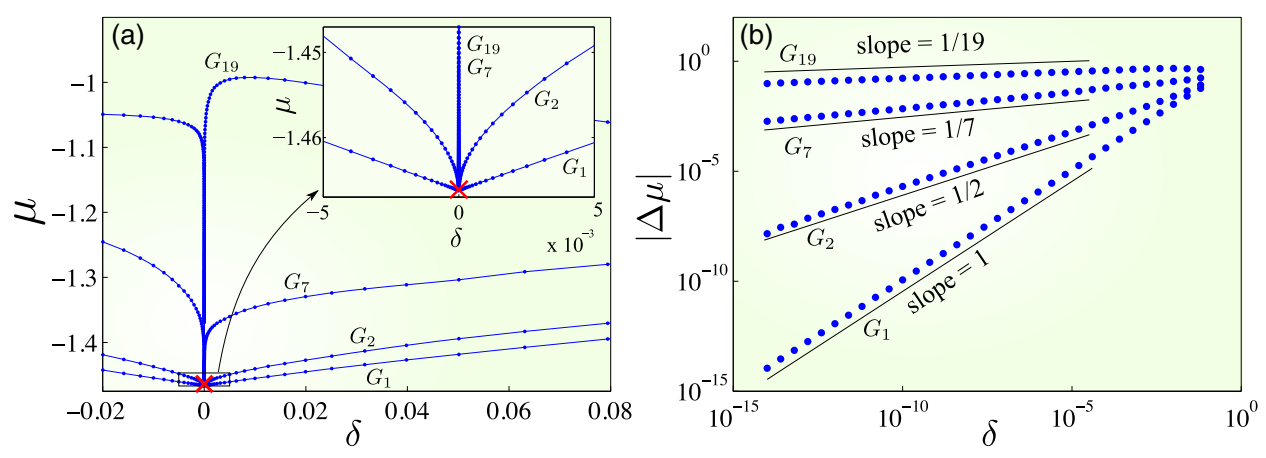

FIG. 9. Sensitivity to weighted perturbations in directed networks of optoelectronic oscillators. (a) Mean convergence rate $\mu$ as a function of $\delta$, illustrating the qualitative difference between sensitive networks $\left(G_{2}, G_{7}\right.$, and $G_{19}$ from Fig. 7) and nonsensitive networks ( $G_{1}$ from Fig. 7). The red cross symbol indicates the value of $\mu$ at $\delta=0$ corresponding to the case of no perturbation, which is the same for all four networks. The inset shows a zoom-in plot of the marked rectangular region surrounding the red cross. The perturbation matrix $\Delta A$ was chosen randomly following the same procedure used in Fig. 7. To facilitate visualization, in this figure we allow negative $\delta$, which corresponds to considering a perturbation term of the form $|\delta|(-\Delta A)$. (b) Log-log plot of the change in convergence rate $|\Delta \mu|$ versus $\delta$, which confirms the scaling $|\Delta \mu| \sim \delta^{1 / \beta}$ for small $\delta$. 
organisms, for example, it is believed that the reason most of their genes are not essential for a given environmental condition is because they are required under different conditions. Bacteria kept under stable conditions, such as those that live inside other living cells (i.e., intracellular bacteria), have evolved to virtually have only those genes essential under that condition [95] and are thus sensitive to gene removals; they are a close analog of the optimization of a fixed objective function considered here [96]. While there is therefore no conflict between our results and the optimization-robustness trade-off expected for biological networks, investigating the equivalent of the sensitive dependence on network structure in the case of varying conditions or varying objective function would likely provide further insights.

In general, the optimization-robustness relation may depend on the type of robustness considered. In this study we focused on how stable a state is, and hence on how resistant the network is to small changes in its dynamical state, which can be regarded as a form of robustness (terminology used, for example, in Ref. [98]). It is quite remarkable that, in seeking to optimize the network for this "dynamical" robustness, the network would lose "structural" robustness, where the latter is a measure of how resistant the stability of the network state is to changes in the network structure. But is the observed sensitive dependence on network structure really a sign of nonrobustness? The answer is both yes and no. It is "yes" in the sense that, because of the nondifferentiability of this dependence, small parameter changes cause stability to change significantly. It is "no" in the sense that, because the cusps appear at valleys rather than at peaks, the stability in the vicinity of the local best parameter choices are still generally better than at locations farther away (that is, specific parameters lead to significant improvement but not to significant deterioration). By considering both the dynamical and the structural robustness in the sense above, we can interpret our results as a manifestation of the "robust-yet-fragile" property that has been suggested as a general feature of complex systems [99].

Finally, it is instructive to compare sensitive dependence on network structure with the phenomenon of chaos, which can exhibit multiple forms of sensitive dependence [100]. Sensitive dependence on initial conditions, where small changes in the initial state lead to large changes in the subsequent evolution of the state, is a phenomenon that concerns trajectories in the phase space of a fixed system. Sensitive dependence on parameters may concern a similar change in trajectories across different systems even when the initial conditions are the same, as in the case of the map $\theta_{n+1}=2 \theta_{n}+c(\bmod 2 \pi)$ when $c$ rather than $\theta_{0}$ is changed. But sensitive dependence on parameters may also concern a change in the nature of the dynamics, which has a qualitative rather than merely quantitative impact on the trajectories; this is the case for the logistic map $x_{n+1}=$ $r x_{n}\left(1-x_{n}\right)$, whose behavior can change from chaotic to periodic by arbitrarily small changes in $r$ and, moreover, whose Lyapunov exponent exhibits a cusplike dependence on $r$ within each periodic window. The latter concerns sensitive dependence of the stability (or the level of stability) of the states under consideration, and therefore is a low-dimensional analog of the sensitive dependence of network dynamics on network structural parameters investigated here. In the case of networks, however, they emerge not from bifurcations but instead from optimization. Much in the same way the discovery of sensitive dependence on initial conditions in the context of (what is now known as) chaos sets constraints on long-term predictability and on the reliability of simple models for weather forecast [101], the sensitive dependence on network structure calls for a careful evaluation of the constraints it sets on predictability and model reliability [102] in the presence of noise and uncertainties in real network systems. We thus believe that the interplay between network structure, optimization, sensitivity, and robustness is a promising topic of future research that can offer fundamental insights into the properties of complex systems.

\section{ACKNOWLEDGMENTS}

This work was supported by the U.S. National Science Foundation under Grant No. DMS-1057128, by the U.S. Army Research Office under Grants No. W911NF-15-10272, No. W911NF-12-1-0276, and No. W911NF-16-10081, and by the Simons Foundation under Grant No. 318812.

\section{APPENDIX A: DERIVATION OF THE STABILITY FUNCTION $\Lambda(\alpha)$}

The two assumptions we make in Sec. II regarding the coupling functions $\mathbf{H}_{i j}$ can be mathematically formulated as follows:

(1) Formulation of assumption (A-1). $D_{\mathbf{u}} \mathbf{H}_{i j}\left(\mathbf{x}^{*}, \mathbf{x}^{*}\right)=$ $-D_{\mathbf{v}} \mathbf{H}_{i j}\left(\mathbf{x}^{*}, \mathbf{x}^{*}\right)$, where $D_{\mathbf{u}} \mathbf{H}_{i j}$ and $D_{\mathbf{v}} \mathbf{H}_{i j}$ denote the derivatives with respect to the first and second argument, respectively, of the function $\mathbf{H}_{i j}(\mathbf{u}, \mathbf{v})$. We also assume $\mathbf{H}_{i j}(\mathbf{u}, \mathbf{u})=0$ for all $\mathbf{u}$, which ensures that the network-homogeneous state is a valid solution of Eq. (1). Together, these assumptions are equivalent to assuming that $\mathbf{H}_{i j}$ can be approximated as $\mathbf{H}_{i j}(\mathbf{u}, \mathbf{v}) \approx D_{\mathbf{v}} \mathbf{H}_{i j}\left(\mathbf{x}^{*}, \mathbf{x}^{*}\right)$. $(\mathbf{v}-\mathbf{u})$ to the first order in $\mathbf{v}-\mathbf{u}$.

(2) Formulation of assumption (A-2). $D_{\mathbf{v}} \mathbf{H}_{i j}\left(\mathbf{x}^{*}, \mathbf{x}^{*}\right)=$ $A_{i j} \cdot \mathbf{G}(t)$, where the scalar $A_{i j}$ is independent of $t$, and the function $\mathbf{G}(t)$ is independent of $i$ and $j$.

Under these assumptions, the variational equation of the system described by Eq. (1) around a given networkhomogeneous state $\mathbf{x}_{1}=\cdots=\mathbf{x}_{n}=\mathbf{x}^{*}(t)$ becomes 


$$
\dot{\boldsymbol{\xi}}_{i}=D_{\mathbf{x}} \mathbf{F} \cdot \boldsymbol{\xi}_{i}-D_{\mathbf{y}} \mathbf{F} \cdot \sum_{j=1}^{n} L_{i j} \mathbf{G}(t) \cdot \boldsymbol{\xi}_{j},
$$

where $\xi_{i}$ is the perturbation to the state of node $i, D_{\mathbf{x}} \mathbf{F}$ and $D_{\mathbf{y}} \mathbf{F}$ are the derivatives of the function $\mathbf{F}$ with respect to the first and any of the other arguments, respectively, evaluated at $\left(\mathbf{x}, \mathbf{y}_{1}, \ldots, \mathbf{y}_{n}\right)=\left(\mathbf{x}^{*}, \mathbf{0}, \ldots, \mathbf{0}\right)$, and $L=\left(L_{i j}\right)$ is the Laplacian matrix of the network given by Eq. (3). An argument based on the Jordan canonical form of $L$ similar to the one used in Ref. [61] then leads to a stability function $\Lambda(\alpha)$, defined for given (complex-valued) auxiliary parameter $\alpha$ as the maximum Lyapunov exponent of the solution $\boldsymbol{\eta}=\mathbf{0}$ of

$$
\dot{\boldsymbol{\eta}}=\left[D_{\mathbf{x}} \mathbf{F}-\alpha D_{\mathbf{y}} \mathbf{F} \cdot \mathbf{G}(t)\right] \boldsymbol{\eta} .
$$

The exponential rate of convergence or divergence is then given by $\Lambda\left(\lambda_{j}\right)$ for the perturbation mode corresponding to the $j$ th (possibly complex) eigenvalue $\lambda_{j}$ of the Laplacian matrix $L$. Thus, the perturbation mode with the slowest convergence (or fastest divergence) determines the stability of the network-homogeneous state through $\Lambda_{\max }$ defined in Eq. (4). A key aspect of this approach is that the functional form of $\Lambda(\alpha)$ does not depend on the network structure, implying that the network structure influences the stability only through the Laplacian eigenvalues [49].

For a system with a global coupling strength parameter $\varepsilon$, such as the networks of identical oscillators and networks of activator-inhibitor systems described in Secs. S1B and S1C of Supplemental Material [58], respectively, the derivative $D_{\mathbf{v}} \mathbf{H}_{i j}\left(\mathbf{x}^{*}, \mathbf{x}^{*}\right)$ in the condition (A-2) above is proportional to $\varepsilon$, and $\mathbf{G}(t)$ can be chosen to include the factor $\varepsilon$ [thus making the stability function $\Lambda(\alpha)=\Lambda_{\varepsilon}(\alpha)$ dependent on $\varepsilon$ ]. We note that the class of systems treated in Ref. [49] is an important special case of our formulation in which $\mathbf{F}\left(\mathbf{x}, \mathbf{y}_{1}, \ldots, \mathbf{y}_{n}\right)$ depends linearly on the $\mathbf{y}$ variables and the coupling function $\mathbf{H}_{i j}$ is proportional to the difference in (some function of) the state of the nodes (details presented in Sec. S1B of Supplemental Material [58]). We also note that the same stability condition $\Lambda_{\max } \leq 0$ is derived in Ref. [56] for a general class of systems that is different from the class of systems treated here. An advantage of our formulation is that the assumptions on the nature of pairwise interactions encoded in the coupling functions $\mathbf{H}_{i j}$ are intuitive and have clear relation to the network structure encoded in the adjacency matrix $A$.

\section{APPENDIX B: UNIQUENESS OF NETWORKS ATTAINING THE BOUND}

Here, we show that, if the mean degree $\bar{d}:=2 \mathrm{~m} / n$ of the network is a (non-negative) integer, the UCM network is the only one that attains the bound in Eq. (8) among all networks with the same $n$ and $m$. For $n=k \ell$ and $m=k^{2} \ell(\ell-1) / 2$, this claim implies that the UCM network is the only $\lambda_{2}$ optimizer. For other combinations of $n$ and $m$, no UCM network exists, and the claim implies that there is no network that can achieve the upper bound.

To prove the claim, we assume that the network attains the bound, i.e., $\lambda_{2}=\lfloor 2 m / n\rfloor=\bar{d}$, and aim to show that it must be a UCM network. We first observe that $\lambda_{n}^{c}=$ $n-\lambda_{2}=n-\bar{d}$. Also, since $\bar{d}$ is an integer, so is the mean degree of the complement, $\bar{d}^{c}=(n-1)-\bar{d}$, and thus Eq. (10) becomes

$$
n-\bar{d}=\lambda_{n}^{c} \geq d_{\max }^{c}+1 \geq \bar{d}^{c}+1=n-\bar{d} .
$$

Since this implies that the maximum and the mean degree of the complement match, i.e., $d_{\max }^{c}=\bar{d}^{c}=: d^{c}$, all nodes must have the same degree $d^{c}$ in the complement. Equation (B1) also implies $\lambda_{n}^{c}=d^{c}+1$. Next we consider an arbitrary connected component of the complement and show that its maximum Laplacian eigenvalue equals $d^{c}+1$. On the one hand, since the Laplacian spectrum of any network is the union of the Laplacian spectra of its connected components (stated and proved as Proposition 3 in Supplemental Material [58], Sec. S2B), we see that the maximum Laplacian eigenvalue of this component is at most $\lambda_{n}^{c}\left(=d^{c}+1\right)$. On the other hand, by applying Eq. (9) to the component and noting that its maximum degree is $d^{c}$, we see that its maximum Laplacian eigenvalue is at least $d^{c}+1$. Combining these, we conclude that the maximum Laplacian eigenvalue of this component equals $d^{c}+1$. We now use the part of Proposition 3.9.3 in Ref. [71] stating that the equality in Eq. (9) holds true only if $d_{\max }+1=n$. Applying this to the component and combining with the result above, we see that the component size must be $k:=d^{c}+1$. Since each node has degree $d^{c}$, the component must be fully connected. Since the choice of the component was arbitrary, the same holds true for all components in the complement, implying that they form $\ell$ isolated, fully connected clusters of size $k$ (for some positive integer $\ell$ ). Therefore, the network must be a UCM network.

\section{APPENDIX C: EXPLICIT CONSTRUCTION OF MCC NETWORKS}

To construct an MCC network for given $n$ and $m$, we first compute the function $M(n, k)$, which we recall is the maximum number of links possible for a network of size $n$ when the largest size of connected components is $\leq k$. For a given $k$, the maximum number of fully connected clusters of size $k$ that one can form with $n$ nodes is $\ell:=$ $\lfloor n / k\rfloor$. Forming $\ell$ such clusters requires $\ell \cdot k(k-1) / 2$ links, and completely connecting the remaining $n_{r}:=$ $n-k \ell$ nodes requires $n_{r}\left(n_{r}-1\right) / 2$ links. Since any 
additional link would necessarily make the size of some component greater than $k$, this network has the maximum possible number of links, and we thus have

$$
M(n, k)=\ell \cdot \frac{k(k-1)}{2}+\frac{n_{r}\left(n_{r}-1\right)}{2}
$$

(proof given in Supplemental Material [58], Sec. S2A). This formula allow us to compute $M(n, k)$ for each $k=1, \ldots, n$. The computed $M(n, k)$ can then be used to determine $k_{n, m_{c}}$ for the given $m$ directly from the definition: $k_{n, m_{c}}$ is the smallest integer $k$ for which $m_{c} \leq M(n, k)$, where $m_{c}=[n(n-1)] / 2-m$.

The complement of an MCC network is then constructed so as to have as many fully connected clusters of size $k_{n, m_{c}}$ as possible using all the $m_{c}$ available links. If one or more links remain, we recursively apply the procedure to these links and the set of remaining isolated nodes. If no cluster of size $k_{n, m_{c}}$ can be formed (which occurs only when $k_{n, m_{c}} \geq 3$ ), we first construct a fully connected cluster of size $k_{n, m_{c}}-1$, which is always possible since $m_{c}>$ $M\left(n, k_{n, m_{c}}-1\right) \geq\left(k_{n, m_{c}}-1\right)\left(k_{n, m_{c}}-2\right) / 2$ by the definition of $k_{n, m_{c}}$. We then connect the remaining links arbitrarily while ensuring that the size of the largest connected component is $k_{n, m_{c}}$. The resulting Laplacian eigenvalues are independent of the configuration of these links, since all possible configurations are equivalent up to permutation of node indices. The procedure thus generates an MCC network with the given number of nodes and links, $n$ and $m$, respectively. Note that, in the special case of $n=k \ell$ and $m=k^{2} \ell(\ell-1) / 2$ with given positive integers $\ell$ and $k$, the procedure we described here results in the UCM network with $\ell$ groups of size $k$, as it is the only MCC network in that case. A MATLAB implementation for the procedure [including the relevant functions such as $M(n, k)$ and $k_{n, m}$ ] is available for download [75].

\section{APPENDIX D: LOWER BOUND FOR MAXIMUM $\operatorname{Re}\left(\lambda_{2}\right)$}

Here, we show that the network constructed in Sec. III B 3 to establish the lower bound satisfies

$$
\begin{aligned}
& \lambda_{1}=0, \quad \lambda_{2}=\cdots=\lambda_{n-r}=s, \\
& \lambda_{n-r+1}=\cdots=\lambda_{n}=s+1,
\end{aligned}
$$

which, in particular, implies that $\lambda_{2}=s$. We first note that $\bar{\lambda}-1<s \leq \bar{\lambda}$, since we have $s=\lfloor\bar{\lambda}\rfloor$ by definition. From the definition of $r$, we can write $r=m_{d}-s(n-1)=$ $\phi n(n-1)-s(n-1)=(\bar{\lambda}-s)(n-1)$. Combining these, we see that $0 \leq r \leq n-2$. We thus divide the proof into two cases: $0 \leq r \leq s-1$ and $s \leq r \leq n-2$. In the following, we use the notation $O_{n_{1} \times n_{2}}$ for the zero matrix of size $n_{1} \times n_{2}$ and $I_{n_{1} \times n_{1}}$ for the identity matrix of size $n_{1}$.
Case 1. If $0 \leq r \leq s-1$, the matrix $L$ has the lower block triangular form

$$
L=\left(\begin{array}{cc}
L_{n_{1} \times n_{1}}^{\prime} & O_{n_{1} \times n_{2}} \\
B_{n_{2} \times n_{1}} & s I_{n_{2} \times n_{2}}
\end{array}\right),
$$

where we use the notations $n_{1}=s+1$ and $n_{2}=n-s-1$. Here, $L_{n_{1} \times n_{1}}^{\prime}$ and $B_{n_{2} \times n_{1}}$ are matrices of size $n_{1} \times n_{1}$ and $n_{2} \times n_{1}$, respectively. The set of eigenvalues of $L$ is thus the union of the set of eigenvalues of $L^{\prime}$ and the set $\{s, \ldots, s\}$ (repeated $n_{2}$ times, owing to the diagonal block $s I_{n_{2} \times n_{2}}$ ). To obtain the eigenvalues of $L_{n_{1} \times n_{1}}^{\prime}$, we apply a sequence of row operations to the matrix $L_{n_{1} \times n_{1}}^{\prime}-\lambda I_{n_{1} \times n_{1}}$. Denoting the $i$ th row of this matrix by $R_{i}$, we first replace $R_{i}$ with $R_{i}-R_{s+1}$ for each $i=1, \ldots, s$, and then replace $R_{s+1}$ with $R_{s+1}+\sum_{i=1}^{r} R_{i} /(s+1-\lambda)+\sum_{i=r+1}^{s} R_{i} /(s-\lambda)$ [or with $R_{s+1}+\sum_{i=1}^{s} R_{i} /(s-\lambda)$, if $\left.r=0\right]$. Because of the specific form of $L_{n_{1} \times n_{1}}^{\prime}-\lambda I_{n_{1} \times n_{1}}$, this results in an upper triangular matrix whose diagonal elements are $s+1-\lambda$ (first $r$ ), $s-\lambda$ (next $s-r$ ), and $-\lambda$. Since none of these row operations involve switching two rows or multiplying a row by a nonzero constant, the determinant is invariant, and hence the eigenvalues of $L_{n_{1} \times n_{1}}^{\prime}$ are $s+1$ (repeated $r$ times), $s$ (repeated $s-r$ times), and 0 (simple). Combining with the $n_{2}$ repetitions of $s$ from the block $s I_{n_{2} \times n_{2}}$ in Eq. (D2), the eigenvalues of $L$ are 0 (simple), $s$ (repeated $s-r+n_{2}=$ $n-r-1$ times), and $s+1$ (repeated $r$ times), satisfying Eq. (D1).

Case 2. If $s \leq r \leq n-2$, the matrix $L$ has the lower block triangular form

$$
L=\left(\begin{array}{ccc}
K_{n_{1} \times n_{1}} & O_{n_{1} \times n_{2}} & O_{n_{1} \times n_{3}} \\
B_{n_{2} \times n_{1}}^{\prime} & (s+1) I_{n_{2} \times n_{2}} & O_{n_{2} \times n_{3}} \\
B_{n_{3} \times n_{1}}^{\prime \prime} & O_{n_{3} \times n_{2}} & s I_{n_{3} \times n_{3}}
\end{array}\right),
$$

where we use the notations $n_{1}=s+1$ and $n_{2}=r-s$, and $n_{3}=n-r-1$. Here, $K_{n_{1} \times n_{1}}$ is the $n_{1} \times n_{1}$ Laplacian matrix of a complete graph of $n_{1}$ nodes, with eigenvalues 0 (simple) and $n_{1}=s+1$ (repeated $n_{1}-1$ times). Therefore, the eigenvalues of matrix $L$ are 0 (simple), $s$ (repeated $n_{3}=n-r-1$ times), and $s+1$ (repeated $n_{1}-1+n_{2}=r$ times), satisfying Eq. (D1).

\section{APPENDIX E: SCALING FOR EIGENVALUES WITH GEOMETRIC DEGENERACY}

We can establish Eq. (19) for an arbitrary eigenvalue of an arbitrary matrix. Given an $n \times n$ matrix $M$, its Jordan decomposition can be written as

$$
M=P J P^{-1}
$$

where $P$ is an invertible matrix and 


$$
J=\left(\begin{array}{ccc}
J^{(1)} & \cdots & 0 \\
\vdots & \ddots & \vdots \\
0 & \cdots & J^{(p)}
\end{array}\right)
$$

is the block-diagonal Jordan matrix with $p$ Jordan blocks [103]. The $j$ th Jordan block is of size $\beta_{j} \times \beta_{j}$ and has the form

$$
J^{(j)}=\left(\begin{array}{ccccc}
\lambda_{j} & 1 & 0 & \cdots & 0 \\
0 & \lambda_{j} & 1 & \cdots & 0 \\
\vdots & \vdots & \ddots & \ddots & \vdots \\
0 & 0 & \cdots & \lambda_{j} & 1 \\
0 & 0 & \cdots & 0 & \lambda_{j}
\end{array}\right)
$$

Since Eq. (E1) is a similarity transformation, the eigenvalues of $M$ are the same as those of $J$, which are the diagonal elements $\lambda_{1}, \lambda_{2}, \ldots, \lambda_{p}$ of $J$ with corresponding multiplicities $\beta_{1}, \beta_{2}, \ldots, \beta_{p}$, respectively. Note that $\beta_{j}$ can be smaller than the algebraic multiplicity of $\lambda_{j}$, since we may have $\lambda_{j}=\lambda_{j^{\prime}}$ for some $j \neq j^{\prime}$.

As in the main text, we consider the matrix perturbation of the form $\hat{M}(\delta)=M+\delta \Delta M$, where $\delta>0$ and $\Delta M$ is an $n \times n$ matrix. For a given eigenvalue $\lambda$ of $M$, let $\alpha$ and $\beta$ denote its algebraic and geometric degeneracy, respectively. The geometric degeneracy is defined as the size of the largest Jordan block associated with $\lambda$, or equivalently, as the largest number of repetitions of $\lambda$ associated with the same eigenvector. Since the roots of a polynomial depend continuously on the coefficients, each eigenvalue of a matrix changes continuously as the elements of that matrix change [104]. Therefore, there are exactly $\alpha$ eigenvalues of the matrix $\hat{M}(\delta)$ that approach $\lambda$ as $\delta \rightarrow 0$. Below, we prove that there exists a constant $C \geq 0$ such that Eq. (19) holds true for each eigenvalue $\hat{\lambda}(\delta)$ of $\hat{M}(\delta)$ that converges to $\lambda$, where we denote $\Delta \lambda(\delta):=\hat{\lambda}(\delta)-\lambda$.

We first use the same $P$ that transforms $M$ into $J$ in Eq. (E1) to transform $\hat{M}(\delta)$ for each $\delta$ as

$$
P^{-1} \hat{M}(\delta) P=J+\delta Q,
$$

where $Q$ is the matrix given by $Q:=P^{-1} \Delta M P$. Thus, the eigenvalues of $\hat{M}(\delta)$ are the same as those of $J+\delta Q$. To further transform the matrix, consider the block-diagonal matrix

$$
T=\left(\begin{array}{ccc}
T^{(1)} & \cdots & 0 \\
\vdots & \ddots & \vdots \\
0 & \cdots & T^{(p)}
\end{array}\right)
$$

where the $j$ th block $T^{(j)}$ is a $\beta_{j} \times \beta_{j}$ diagonal matrix with elements $T_{i i}^{(j)}=\delta^{-1+i / \beta_{j}}, 1 \leq i \leq \beta_{j}$. The matrix $T$ is invertible for all $\delta \neq 0$. Therefore, the eigenvalues of $\hat{M}(\delta)$ are the same as those of the matrix

$$
T^{-1} P^{-1} \hat{M}(\delta) P T=T^{-1} J T+\delta T^{-1} Q T .
$$

From the definition of $T$, it follows that the matrix $T^{-1} J T$ has the same block-diagonal structure as $J$ and $T$, and the $j$ th diagonal block is the matrix

$$
\left(\begin{array}{ccccc}
\lambda_{j} & \delta^{1 / \beta_{j}} & 0 & \cdots & 0 \\
0 & \lambda_{j} & \delta^{1 / \beta_{j}} & \cdots & 0 \\
\vdots & \vdots & \ddots & \ddots & \vdots \\
0 & 0 & \cdots & \lambda_{j} & \delta^{1 / \beta_{j}} \\
0 & 0 & \cdots & 0 & \lambda_{j}
\end{array}\right) .
$$

It also follows that the $(i, k)$ element of the matrix $\delta T^{-1} Q T$ is upper bounded by $\left|Q_{i k}\right| \delta^{1 / \beta_{j}}$, where $j$ is the index for the Jordan block that intersects with the $k$ th column of the matrix $J$. Applying the Gershgorin theorem [104] to the right-hand side of Eq. (E6), we see that each eigenvalue of $\hat{M}(\delta)$ must be contained in the disk centered at $\lambda_{j}$ with radius $C \delta^{1 / \beta_{j}}$ for some $j=1, \ldots, p$, where $C:=1+\max _{k} \sum_{i}\left|Q_{i k}\right|$. [The first term in the expression for $C$ comes from the offdiagonal elements in Eq. (E7).]

Now the algebraic and geometric multiplicity of the given eigenvalue $\lambda$ of $M$ can be expressed as $\alpha=\sum_{j} \beta_{j}$ and $\beta=\max _{j} \beta_{j}$, respectively, where the sum and the maximum are both taken over all $j$ for which $\lambda_{j}=\lambda$. Choose $\hat{\lambda}(\delta)$ to be any of the $\alpha$ eigenvalues of $\hat{M}(\delta)$ that converge to $\lambda$ as $\delta \rightarrow 0$. Also choose a fixed $\delta$ value sufficiently small to ensure that any two disks with different centers among those mentioned above in connection with the Gershgorin theorem are disjoint (which can be achieved if $\max _{j} C \delta^{1 / \beta_{j}}$ is less than half the minimum distance between distinct eigenvalues of $M$ ). With this choice, the disk centered at $\lambda$ with radius $C \delta^{1 / \beta}$ is disjoint from all the others and must contain $\hat{\lambda}(\delta)$; otherwise, $\hat{\lambda}(\delta)$ would have to jump discontinuously from another disk as $\delta \rightarrow 0$ since it must remain in at least one of these disks, and this would violate the continuity of $\hat{\lambda}(\delta)$ with respect to $\delta$. Having $\hat{\lambda}(\delta)$ in the disk centered at $\lambda$ with radius $C \delta^{1 / \beta}$ immediately gives the inequality in Eq. (19).

\section{APPENDIX F: NONSENSITIVITY UNDER WEIGHTED CONSTRAINED PERTURBATIONS}

We can show that all eigenvalues are nonsensitive under a certain class of weighted perturbations even when $\beta>1$. If 
the matrix $P$ for the Jordan decomposition of $M$ in Eq. (E1) transforms the perturbation matrix $\Delta M$ into an upper triangular matrix, then the matrix $T^{-1} Q T$ in Eq. (E6) is also upper triangular. In this case, we have the stronger result that the perturbed eigenvalues are given precisely by $\hat{\lambda}(\delta)=\lambda+\delta Q_{i i}$, where $i$ is the index for any column of $J$ that intersects with a Jordan block associated with the eigenvalue $\lambda$. The change of each eigenvalue is thus proportional to $\delta$, i.e., the scaling exponent is one, independently of $\beta$ [which is consistent with the general result in Eq. (19) since $\beta \geq 1$ ]. The result for nongeneric perturbations in Sec. IVA 2 follows from this if $M$ is replaced by the Laplacian matrix $L$ and $\Delta M$ by $\Delta L$. In particular, the result applies to the case of a directed tree with each link having equal weight and $\Delta L$ representing a perturbation of the weights of the existing links.

\section{APPENDIX G: SCALING FOR APPROXIMATELY DEGENERATE NETWORKS}

Here, we show that the scaling in Eqs. (19) and (20) is observed even when the eigenvector is only approximately degenerate. More precisely, we show that, when the matrix is close to one with exact degeneracy, the scaling remains valid over a range of $\delta$ much larger than the distance between the two matrices.

Suppose that a matrix $M_{0}$ has an eigenvalue $\lambda\left(M_{0}\right)$ with exact geometric degeneracy $\beta$. We consider a perturbation of $M_{0}$ in the form $M_{1}=M_{0}+\varepsilon \Delta M_{1}$, where $\Delta M_{1}$ is a fixed matrix satisfying $\left\|\Delta M_{1}\right\|=1$. Thus, the distance between $M_{0}$ and $M_{1}$ is $\varepsilon$, and for small $\varepsilon$ (and a generic choice of $\Delta M_{1}$ ) the matrix $M_{1}$ is approximately degenerate. We now apply a perturbation of size $\delta$ to $M_{1}$ in the form $M_{2}=M_{1}+\delta \Delta M_{2}$, where $\Delta M_{2}$ is another fixed matrix satisfying $\left\|\Delta M_{2}\right\|=1$. Denoting $\eta:=\varepsilon / \delta$, we can write $M_{2}$ as a perturbation of $M_{0}$ rather than $M_{1}$; namely, $M_{2}=M_{0}+\delta\left(\eta \Delta M_{1}+\Delta M_{2}\right)$.

When taking the limit $\delta \rightarrow 0$ with $\eta$ fixed, matrices $\Delta M_{1}$ and $\eta \Delta M_{1}+\Delta M_{2}$ are both fixed, so we can apply the result in Eq. (19). We thus have

$$
\begin{gathered}
\limsup _{\delta \rightarrow 0} \frac{\left|\lambda\left(M_{1}\right)-\lambda\left(M_{0}\right)\right|}{(\eta \delta)^{1 / \beta}} \leq C_{1}, \\
\limsup _{\delta \rightarrow 0} \frac{\left|\lambda\left(M_{2}\right)-\lambda\left(M_{0}\right)\right|}{\delta^{1 / \beta}} \leq C_{2},
\end{gathered}
$$

for some constants $C_{1}, C_{2} \geq 0$, where $\lambda\left(M_{1}\right)$ and $\lambda\left(M_{2}\right)$ denote eigenvalues of $M_{1}$ and $M_{2}$, respectively, that approach $\lambda\left(M_{0}\right)$ as $\delta \rightarrow 0$. This means that for an arbitrary $\xi>0$, we can find $\delta_{1}>0$ and $\delta_{2}>0$ (which can depend on $\eta$ ) such that

$$
\begin{aligned}
& \frac{\left|\lambda\left(M_{1}\right)-\lambda\left(M_{0}\right)\right|}{(\eta \delta)^{1 / \beta}}<C_{1}+\frac{\xi}{2 \eta^{1 / \beta}}, \quad \text { if } \delta<\delta_{1}, \\
& \frac{\left|\lambda\left(M_{2}\right)-\lambda\left(M_{0}\right)\right|}{\delta^{1 / \beta}}<C_{2}+\frac{\xi}{2}, \quad \text { if } \delta<\delta_{2} .
\end{aligned}
$$

Then,

$$
\begin{aligned}
\frac{\left|\lambda\left(M_{2}\right)-\lambda\left(M_{1}\right)\right|}{\delta^{1 / \beta}} & =\frac{\left|\left[\lambda\left(M_{2}\right)-\lambda\left(M_{0}\right)\right]-\left[\lambda\left(M_{1}\right)-\lambda\left(M_{0}\right)\right]\right|}{\delta^{1 / \beta}} \\
& \leq \frac{\left|\lambda\left(M_{2}\right)-\lambda\left(M_{0}\right)\right|}{\delta^{1 / \beta}}+\frac{\left|\lambda\left(M_{1}\right)-\lambda\left(M_{0}\right)\right|}{(\eta \delta)^{1 / \beta}} \cdot \eta^{1 / \beta} \\
& <\left(C_{2}+\frac{\xi}{2}\right)+\left(C_{1}+\frac{\xi}{2 \eta^{1 / \beta}}\right) \cdot \eta^{1 / \beta} \\
& =C_{2}+C_{1} \eta^{1 / \beta}+\xi, \quad(\mathrm{G} 3)
\end{aligned}
$$

if $\delta<\min \left(\delta_{1}, \delta_{2}\right)$. Since $\xi$ can be made arbitrarily small by making $\delta$ sufficiently small, we have

$$
\underset{\delta \rightarrow 0}{\limsup } \frac{\left|\lambda\left(M_{2}\right)-\lambda\left(M_{1}\right)\right|}{\delta^{1 / \beta}} \leq C_{2}+C_{1} \eta^{1 / \beta} .
$$

Thus, Eq. (19) and the corresponding bound on the scaling exponent, $\gamma \geq 1 / \beta$, remain valid for any fixed $\eta$ (i.e., with $\varepsilon \rightarrow 0$ as $\delta \rightarrow 0$ while holding $\eta=\varepsilon / \delta$ constant). For finite $\varepsilon$ and $\delta$, this result suggests that we should observe the scaling $\left|\lambda\left(M_{2}\right)-\lambda\left(M_{1}\right)\right| \sim \delta^{\gamma}$ with $\gamma \geq 1 / \beta$ when $\varepsilon \ll \delta \ll 1$.

Now consider the stronger scaling property in Eq. (20), which can be formalized for $M_{1}$ and $M_{2}$ as

$\lim _{\delta \rightarrow 0} \frac{\left|\lambda\left(M_{1}\right)-\lambda\left(M_{0}\right)\right|}{(\eta \delta)^{1 / \beta}}=C_{1}, \quad \lim _{\delta \rightarrow 0} \frac{\left|\lambda\left(M_{2}\right)-\lambda\left(M_{0}\right)\right|}{\delta^{1 / \beta}}=C_{2}$.

Replacing Eq. (G1) with Eq. (G5) and using the resulting lower bounds analogous to those in Eq. (G2), we obtain a lower bound analogous to that in Eq. (G3). Combining this with Eq. (G3), we obtain

$$
\left|\frac{\left|\lambda\left(M_{2}\right)-\lambda\left(M_{1}\right)\right|}{\delta^{1 / \beta}}-C_{2}\right|<C_{1} \eta^{1 / \beta}+\xi .
$$

Since $\xi$ can be made arbitrarily small by making $\delta$ sufficiently small, we see that

$$
\underset{\delta \rightarrow 0}{\limsup }\left|\frac{\left|\lambda\left(M_{2}\right)-\lambda\left(M_{1}\right)\right|}{\delta^{1 / \beta}}-C_{2}\right| \leq C_{1} \eta^{1 / \beta} .
$$

This implies the scaling in Eq. (20), or more precisely, $\left|\lambda\left(M_{2}\right)-\lambda\left(M_{1}\right)\right|=C \delta^{1 / \beta}$ with a prefactor $C$ that can vary with $\delta$ but is bounded between $C_{2} \pm C_{1} \eta^{1 / \beta}$ as $\delta \rightarrow 0$. The ratio $\eta$ of perturbation sizes thus determines the range of variation of this scaling prefactor. In the limit of both $\eta \rightarrow 0$ and $\delta \rightarrow 0$, Eq. (G6) implies 
$\lim _{\eta, \delta \rightarrow 0}\left|\lambda\left(M_{2}\right)-\lambda\left(M_{1}\right)\right| / \delta^{1 / \beta}=C_{2}$. Therefore, we have the scaling $\left|\lambda\left(M_{2}\right)-\lambda\left(M_{1}\right)\right| \approx C_{2} \delta^{1 / \beta}$ when $\varepsilon \ll \delta \ll 1$.

Altogether, we have shown that the scaling properties in Eqs. (19) and (20) are observed for the eigenvalues of $M_{2}$ when the size $\delta$ of the perturbation applied to $M_{1}$ is much larger compared to the distance $\varepsilon$ between $M_{1}$ and the exactly degenerate matrix $M_{0}$.

[1] M. E. J. Newman, Networks: An Introduction (Oxford University Press, Oxford, England, 2010).

[2] G. Chen, X. Wang, and X. Li, Fundamentals of Complex Networks: Models, Structures and Dynamics (John Wiley and Sons, Singapore, 2015).

[3] S. H. Strogatz, Exploring Complex Networks, Nature (London) 410, 268 (2001).

[4] A. Barrat, M. Barthélemy, and A. Vespignani, Dynamical Processes on Complex Networks (Cambridge University Press, Cambridge, England, 2008).

[5] M. A. Porter and J. P. Gleeson, Dynamical Systems on Networks (Springer International Publishing, 2016).

[6] T. Nishikawa, A. E. Motter, Y.C. Lai, and F. C. Hoppensteadt, Heterogeneity in Oscillator Networks: Are Smaller Worlds Easier to Synchronize?, Phys. Rev. Lett. 91, 014101 (2003).

[7] J. G. Restrepo, E. Ott, and B. R. Hunt, Spatial Patterns of Desynchronization Bursts in Networks, Phys. Rev. E 69, 066215 (2004).

[8] H. Kori and A. S. Mikhailov, Entrainment of Randomly Coupled Oscillator Networks by a Pacemaker, Phys. Rev. Lett. 93, 254101 (2004).

[9] I. Belykh, V. Belykh, and M. Hasler, Generalized Connection Graph Method for Synchronization in Asymmetrical Networks, Physica (Amsterdam) 224D, 42 (2006).

[10] J. G. Restrepo, E. Ott, and B. R. Hunt, Emergence of Coherence in Complex Networks of Heterogeneous Dynamical Systems, Phys. Rev. Lett. 96, 254103 (2006).

[11] D. A. Wiley, S. H. Strogatz, and M. Girvan, The Size of the Sync Basin, Chaos 16, 015103 (2006).

[12] H. Kori and A. S. Mikhailov, Strong Effects of Network Architecture in the Entrainment of Coupled Oscillator Systems, Phys. Rev. E 74, 066115 (2006).

[13] A. Arenas, A. Díaz-Guilera, J. Kurths, Y. Moreno, and C. Zhou, Synchronization in Complex Networks, Phys. Rep. 469, 93 (2008).

[14] V. Nicosia, M. Valencia, M. Chavez, A. Díaz-Guilera, and V. Latora, Remote Synchronization Reveals Network Symmetries and Functional Modules, Phys. Rev. Lett. 110, 174102 (2013).

[15] L. M. Pecora, F. Sorrentino, A. M. Hagerstrom, T. E. Murphy, and R. Roy, Cluster Synchronization and Isolated Desynchronization in Complex Networks with Symmetries, Nat. Commun. 5, 4079 (2014).

[16] P. S. Skardal, D. Taylor, and J. Sun, Optimal Synchronization of Complex Networks, Phys. Rev. Lett. 113, 144101 (2014).
[17] V. Colizza, R. Pastor-Satorras, and A. Vespignani, Reaction-Diffusion Processes and Metapopulation Models in Heterogeneous Networks, Nat. Phys. 3, 276 (2007).

[18] S. Gómez, A. Díaz-Guilera, J. Gómez-Gardeñes, C. J. PérezVicente, Y. Moreno, and A. Arenas, Diffusion Dynamics on Multiplex Networks, Phys. Rev. Lett. 110, 028701 (2013).

[19] M. Youssef, Y. Khorramzadeh, and S. Eubank, Network Reliability: The Effect of Local Network Structure on Diffusive Processes, Phys. Rev. E 88, 052810 (2013).

[20] S. Hata, H. Nakao, and A. S. Mikhailov, Advection of Passive Particles over Flow Networks, Phys. Rev. E 89, 020801 (2014).

[21] A. Pomerance, E. Ott, M. Girvan, and W. Losert, The Effect of Network Topology on the Stability of Discrete State Models of Genetic Control, Proc. Natl. Acad. Sci. U.S.A. 106, 8209 (2009).

[22] L. A. Bunimovich and B.Z. Webb, Isospectral Graph Transformations, Spectral Equivalence, and Global Stability of Dynamical Networks, Nonlinearity 25, 211 (2012).

[23] F. Sorrentino, M. di Bernardo, F. Garofalo, and G. Chen, Controllability of Complex Networks via Pinning, Phys. Rev. E 75, 046103 (2007).

[24] A. J. Whalen, S. N. Brennan, T. D. Sauer, and S. J. Schiff, Observability and Controllability of Nonlinear Networks: The Role of Symmetry, Phys. Rev. X 5, 011005 (2015).

[25] S. Sreenivasan, R. Cohen, E. López, Z. Toroczkai, and H. E. Stanley, Structural Bottlenecks for Communication in Networks, Phys. Rev. E 75, 036105 (2007).

[26] J. Sun, D. Taylor, and E. M. Bollt, Causal Network Inference by Optimal Causation Entropy, SIAM J. Appl. Dyn. Syst. 14, 73 (2015).

[27] C. Fu, Z. Deng, L. Huang, and X. Wang, Topological Control of Synchronous Patterns in Systems of Networked Chaotic Oscillators, Phys. Rev. E 87, 032909 (2013).

[28] Y. Qian, X. Huang, G. Hu, and X. Liao, Structure and Control of Self-Sustained Target Waves in Excitable Small-World Networks, Phys. Rev. E 81, 036101 (2010).

[29] T. Gross, C. J. D. D'Lima, and B. Blasius, Epidemic Dynamics on an Adaptive Network, Phys. Rev. Lett. 96, 208701 (2006).

[30] S. Risau-Gusman and D. H. Zanette, Contact Switching as a Control Strategy for Epidemic Outbreaks, J. Theor. Biol. 257, 52 (2009).

[31] A. Hagberg and D. A. Schult, Rewiring Networks for Synchronization, Chaos 18, 037105 (2008).

[32] T. Nishikawa and A. E. Motter, Network Synchronization Landscape Reveals Compensatory Structures, Quantization, and the Positive Effect of Negative Interactions, Proc. Natl. Acad. Sci. U.S.A. 107, 10342 (2010).

[33] T. Watanabe and N. Masuda, Enhancing the Spectral Gap of Networks by Node Removal, Phys. Rev. E 82, 046102 (2010).

[34] J. D. Hart, J. P. Pade, T. Pereira, T. E. Murphy, and R. Roy, Adding Connections Can Hinder Network Synchronization of Time-Delayed Oscillators, Phys. Rev. E 92, 022804 (2015).

[35] N. A. M. Araújo, H. Seybold, R. M. Baram, H. J. Herrmann, and J. S. Andrade, Optimal Synchronizability of Bearings, Phys. Rev. Lett. 110, 064106 (2013). 
[36] B. Ravoori, A. B. Cohen, J. Sun, A. E. Motter, T. E. Murphy, and R. Roy, Robustness of Optimal Synchronization in Real Networks, Phys. Rev. Lett. 107, 034102 (2011).

[37] M. Nixon, M. Fridman, E. Ronen, A. A. Friesem, N. Davidson, and I. Kanter, Controlling Synchronization in Large Laser Networks, Phys. Rev. Lett. 108, 214101 (2012).

[38] I. Dobson, B. A. Carreras, V. E. Lynch, and D. E. Newman, Complex Systems Analysis of Series of Blackouts: Cascading Failure, Critical Points, and Self-Organization, Chaos 17, 026103 (2007).

[39] D. L. K. Yamins, H. Hong, C. F. Cadieu, E. A. Solomon, D. Seibert, and J. J. DiCarlo, Performance-Optimized Hierarchical Models Predict Neural Responses in Higher Visual Cortex, Proc. Natl. Acad. Sci. U.S.A. 111, 8619 (2014).

[40] G. Buzsáki, C. Geisler, D. A. Henze, and X. J. Wang, Interneuron Diversity Series: Circuit Complexity and Axon Wiring Economy of Cortical Interneurons, Trends Neurosci. 27, 186 (2004).

[41] G. Tononi, O. Sporns, and G. M. Edelman, A Measure for Brain Complexity: Relating Functional Segregation and Integration in the Nervous System, Proc. Natl. Acad. Sci. U.S.A. 91, 5033 (1994).

[42] E. Bullmore and O. Sporns, Complex Brain Networks: Graph Theoretical Analysis of Structural and Functional Systems, Nat. Rev. Neurosci. 10, 186 (2009).

[43] S. Achard and E. Bullmore, Efficiency and Cost of Economical Brain Functional Networks, PLoS Comput. Biol. 3, e17 (2007).

[44] G. H. Golub and C. F. Van Loan, Matrix Computations (The Johns Hopkins University Press, Baltimore, 2013).

[45] M. Eslami, Theory of Sensitivity in Dynamic Systems (Springer-Verlag, Berlin, 1994).

[46] C. K. Chui and G. Chen, Discrete $H^{\infty}$ Optimization (Springer-Verlag, Berlin, 1997).

[47] B. Ottino-Löffler and S.H. Strogatz, Comparing the Locking Threshold for Rings and Chains of Oscillators, Phys. Rev. E 94, 062203 (2016).

[48] B. D. MacArthur and R. J. Sánchez-García, Spectral Characteristics of Network Redundancy, Phys. Rev. E 80, 026117 (2009).

[49] L. M. Pecora and T. L. Carroll, Master Stability Functions for Synchronized Coupled Systems, Phys. Rev. Lett. 80, 2109 (1998).

[50] W. Ren, R. Beard, and E. Atkins, Information Consensus in Multivehicle Cooperative Control, IEEE Control Syst. Mag. 27, 71 (2007).

[51] P. Yang, R. Freeman, and K. Lynch, Multi-Agent Coordination by Decentralized Estimation and Control, IEEE Trans. Autom. Control 53, 2480 (2008).

[52] H. Nakao and A.S. Mikhailov, Turing Patterns in Network-Organized Activator-Inhibitor Systems, Nat. Phys. 6, 544 (2010).

[53] C. Maas, Transportation in Graphs and the Admittance Spectrum, Discrete Appl. Math. 16, 31 (1987).

[54] A. E. Motter, S. A. Myers, M. Anghel, and T. Nishikawa, Spontaneous Synchrony in Power-Grid Networks, Nat. Phys. 9, 191 (2013).

[55] Y. Kuramoto, Chemical Oscillations, Waves, and Turbulence (Springer-Verlag, Berlin, 1984).
[56] K. S. Fink, G. Johnson, T. Carroll, D. Mar, and L. Pecora, Three Coupled Oscillators as a Universal Probe of Synchronization Stability in Coupled Oscillator Arrays, Phys. Rev. E 61, 5080 (2000).

[57] T. Nishikawa and A. E. Motter, Synchronization is Optimal in Non-Diagonalizable Networks, Phys. Rev. E 73, 065106 (2006).

[58] See Supplemental Material at http://link.aps.org/ supplemental/10.1103/PhysRevX.7.041044 for the description of several example systems and processes (Sec. S1), proofs of key properties of the MCC networks (Sec. S2), and supplemental figures (Figs. S1-S3).

[59] J. Sun, E. M. Bollt, and T. Nishikawa, Master Stability Functions for Coupled Nearly Identical Dynamical Systems, Europhys. Lett. 85, 60011 (2009).

[60] L. Donetti, P. I. Hurtado, and M. A. Muñoz, Entangled Networks, Synchronization, and Optimal Network Topology, Phys. Rev. Lett. 95, 188701 (2005).

[61] T. Nishikawa and A.E. Motter, Maximum Performance at Minimum Cost in Network Synchronization, Physica (Amsterdam) 224D, 77 (2006).

[62] B. Wang, T. Zhou, Z. L. Xiu, and B. J. Kim, Optimal Synchronizability of Networks, Eur. Phys. J. B 60, 89 (2007).

[63] M. Brede, Optimal Synchronization in Space, Phys. Rev. E 81, 025202 (2010).

[64] Q. Xinyun, W. Lifu, G. Yuan, and W. Yaping, in Proceedings of the 25th Chinese Control and Decision Conference (IEEE, Guiyang, China, 2013), p. 3414.

[65] N. Alon, Eigenvalues and Expanders, Combinatorica 6, 83 (1986).

[66] A. Lubotzky, R. Phillips, and P. Sarnak, Ramanujan Graphs, Combinatorica 8, 261 (1988).

[67] J. Friedman, J. Kahn, and E. Szemerédi, in Proceedings of the Twenty-First Annual ACM Symposium on Theory of Computing (STOC '89), edited by D. S. Johnson (ACM, New York, 1989), p. 587.

[68] T. Kolokolnikov, Maximizing Algebraic Connectivity for Certain Families of Graphs, Linear Algebra Appl. 471, 122 (2015).

[69] R. Diestel, Graph Theory (Springer-Verlag, Berlin, 2005).

[70] Z. Duan, C. Liu, and G. Chen, Network Synchronizability Analysis: The Theory of Subgraphs and Complementary Graphs, Physica (Amsterdam) 237D, 1006 (2008).

[71] A. Brouwer, Spectra of Graphs (Springer, New York, 2012).

[72] R. Milo, S. Shen-Orr, S. Itzkovitz, N. Kashtan, D. Chklovskii, and U. Alon, Network Motifs: Simple Building Blocks of Complex Networks, Science 298, 824 (2002).

[73] O. Sporns and R. Kötter, Motifs in Brain Networks, PLoS Biol. 2, e369 (2004).

[74] P. Kaluza, M. Ipsen, M. Vingron, and A. S. Mikhailov, Design and Statistical Properties of Robust Functional Networks: A Model Study of Biological Signal Transduction, Phys. Rev. E 75, 015101 (2007).

[75] https://github.com/tnishi0/mcc-networks.

[76] D. Achlioptas, R. M. D'Souza, and J. Spencer, Explosive Percolation in Random Networks, Science 323, 1453 (2009).

[77] R. A. da Costa, S. N. Dorogovtsev, A. V. Goltsev, and J.F.F. Mendes, Explosive Percolation Transition Is Actually Continuous, Phys. Rev. Lett. 105, 255701 (2010). 
[78] O. Riordan and L. Warnke, Explosive Percolation Is Continuous, Science 333, 322 (2011).

[79] J. Nagler, A. Levina, and M. Timme, Impact of Single Links in Competitive Percolation, Nat. Phys. 7, 265 (2011).

[80] R. M. D'Souza and J. Nagler, Anomalous Critical and Supercritical Phenomena in Explosive Percolation, Nat. Phys. 11, 531 (2015).

[81] H. Rozenfeld, L. Gallos, and H. Makse, Explosive Percolation in the Human Protein Homology Network, Eur. Phys. J. B 75, 305 (2010).

[82] W. Chen, M. Schröder, R. M. D’Souza, D. Sornette, and J. Nagler, Microtransition Cascades to Percolation, Phys. Rev. Lett. 112, 155701 (2014).

[83] We note that directed networks can exhibit sensitive dependence on the network structure with respect to different objective functions (see, e.g., Ref. [32]).

[84] A. F. Taylor, M. R. Tinsley, and K. Showalter, Insights into Collective Cell Behaviour from Populations of Coupled Chemical Oscillators, Phys. Chem. Chem. Phys. 17, 20047 (2015).

[85] I. Z. Kiss, Y. Zhai, and J. L. Hudson, Emerging Coherence in a Population of Chemical Oscillators, Science 296, 1676 (2002).

[86] MATLAB, version 7.10.0 (R2010a), The MathWorks Inc., 2010.

[87] G. Golub and C. Van Loan, Matrix Computations (Johns Hopkins University Press, Baltimore, 2013).

[88] R. A. Brualdi and D. Cvetkovic, A Combinatorial Approach to Matrix Theory and Its Applications (CRC Press, Boca Raton, FL, 2008).

[89] L. Trefethen and M. Embree, Spectra and Pseudospectra: The Behavior of Nonnormal Matrices and Operators (Princeton University Press, Princeton, NJ, 2005).

[90] A. Milanese, J. Sun, and T. Nishikawa, Approximating Spectral Impact of Structural Perturbations in Large Networks, Phys. Rev. E 81, 046112 (2010).

[91] H. Kitano, Biological Robustness, Nat. Rev. Genet. 5, 826 (2004).

[92] T. Baba, T. Ara, M. Hasegawa, Y. Takai, Y. Okumura, M. Baba, K. A. Datsenko, M. Tomita, B. L. Wanner, and
H. Mori, Construction of Escherichia coli K-12 In-Frame, Single-Gene Knockout Mutants: The Keio Collection, Mol. Syst. Biol. 2, 2006.0008 (2006).

[93] J. Stelling, U. Sauer, Z. Szallasi, F. J. Doyle III, and J. Doyle, Robustness of Cellular Functions, Cell 118, 675 (2004).

[94] L. Yang, S. Srinivasan, R. Mahadevan, and W. R. Cluett, Characterizing Metabolic Pathway Diversification in the Context of Perturbation Size, Metab. Eng. 28, 114 (2015).

[95] J. I. Glass, N. Assad-Garcia, N. Alperovich, S. Yooseph, M. R. Lewis, M. Maruf, C. A. Hutchison, H. O. Smith, and J.C. Venter, Essential Genes of a Minimal Bacterium, Proc. Natl. Acad. Sci. U.S.A. 103, 425 (2006).

[96] A complementary example of interplay between selection optimization and robustness is described in Ref. [97], which shows that optimization can drive a population to extinction through a global bifurcation in the structure of the state space.

[97] K. Parvinen and U. Dieckmann, Self-Extinction through Optimizing Selection, J. Theor. Biol. 333, 1 (2013).

[98] Y. Bar-Yam and I. R. Epstein, Response of Complex Networks to Stimuli, Proc. Natl. Acad. Sci. U.S.A. 101, 4341 (2004).

[99] J. M. Carlson and J. Doyle, Highly Optimized Tolerance: A Mechanism for Power Laws in Designed Systems, Phys. Rev. E 60, 1412 (1999).

[100] E. Ott, Chaos in Dynamical Systems, 2nd ed. (Cambridge University Press, Cambridge, England, 2002).

[101] E. N. Lorenz, Deterministic Nonperiodic Flow, J. Atmos. Sci. 20, 130 (1963).

[102] A. C. Babtie, P. Kirk, and M. P. H. Stumpf, Topological Sensitivity Analysis for Systems Biology, Proc. Natl. Acad. Sci. U.S.A. 111, 18507 (2014).

[103] A. F. Filippov, A Short Proof of the Theorem on Reduction of a Matrix to Jordan Form, Vestnik Moskov. Univ. Ser. I Mat. Meh. 26, 18 (1971).

[104] R. A. Horn and C. R. Johnson, Matrix Analysis (Cambridge University Press, Cambridge, England, 1985). 